EUROPEAN ORGANIZATION FOR NUCLEAR RESEARCH

CERN-EP-2000-070

31 May 2000

\title{
Multiplicities of $\pi^{0}, \eta, \quad \mathbf{K}^{0}$ and of charged particles in quark and gluon jets
}

\author{
The OPAL Collaboration
}

\begin{abstract}
We compared the multiplicities of $\pi^{0}, \eta, \mathrm{K}^{0}$ and of charged particles in quark and gluon jets in 3-jet events, as measured by the OPAL experiment at LEP. The comparisons were performed for distributions unfolded to $100 \%$ pure quark and gluon jets, at an effective scale $Q_{j \text { jet }}$ which took into account topological dependences of the 3 -jet environment. The ratio of particle multiplicity in gluon jets to that in quark jets as a function of $\mathrm{Q}_{\text {jet }}$ for $\pi^{0}, \eta$ or $\mathrm{K}^{0}$ was found to be independent of the particle species. This is consistent with the QCD prediction that the observed enhancement in the mean particle rate in gluon jets with respect to quark jets should be independent of particle species. In contrast to some theoretical predictions and previous observations, we observed no evidence for an enhancement of $\eta$ meson production in gluon jets with respect to quark jets, beyond that observed for charged particles. We measured the ratio of the slope of the average charged particle multiplicity in gluon jets to that in quark jets, $\mathrm{C}$, and we compared it to a next-to-next-to-next-to leading order calculation. Our result, $\mathrm{C}=2.27 \pm 0.20$ (stat + syst), is about one standard deviation higher than the perturbative prediction.
\end{abstract}

(Submitted to Eur. Phys. Jour. C) 


\section{The OPAL Collaboration}

G. Abbiendi ${ }^{2}$, K. Ackerstaff ${ }^{8}$, C. Ainsley ${ }^{5}$, P.F. Akesson ${ }^{3}$, G. Alexander ${ }^{22}$, J. Allison ${ }^{16}$, K.J. Anderson ${ }^{9}$, S. Arcelli ${ }^{17}$, S. Asai ${ }^{23}$, S.F. Ashby ${ }^{1}$, D. Axen ${ }^{27}$, G. Azuelos ${ }^{18, a}$, I. Bailey ${ }^{26}$, A.H. Ball ${ }^{8}$, E. Barberio ${ }^{8}$, R.J. Barlow ${ }^{16}$, J.R. Batley ${ }^{5}$, S. Baumann ${ }^{3}$, T. Behnke ${ }^{25}$, K.W.Bell ${ }^{20}$, G. Bella ${ }^{22}$, A. Bellerive ${ }^{9}$, S. Bentvelsen ${ }^{8}$, S. Bethke ${ }^{14, i}$, O. Biebel ${ }^{14, i}$, I.J. Bloodworth ${ }^{1}$, P. Bock ${ }^{11}$, J. Böhme ${ }^{14, h}$, O. Boeriu ${ }^{10}$, D. Bonacorsi ${ }^{2}$, M. Boutemeur ${ }^{31}$,

S. Braibant ${ }^{8}$, P. Bright-Thomas ${ }^{1}$, L. Brigliadori ${ }^{2}$, R.M. Brown ${ }^{20}$, H.J. Burckhart ${ }^{8}$,

J. Cammin ${ }^{3}$, P. Capiluppi ${ }^{2}$, R.K. Carnegie ${ }^{6}$, A.A. Carter ${ }^{13}$, J.R. Carter ${ }^{5}$, C.Y. Chang ${ }^{17}$,

D.G. Charlton ${ }^{1, b}$, C. Ciocca ${ }^{2}$, P.E.L. Clarke ${ }^{15}$, E. Clay ${ }^{15}$, I. Cohen ${ }^{22}$, O.C. Cooke ${ }^{8}$, J. Couchman ${ }^{15}$, C. Couyoumtzelis ${ }^{13}$, R.L. Coxe ${ }^{9}$, M. Cuffiani ${ }^{2}$, S. Dado ${ }^{21}$, G.M. Dallavalle ${ }^{2}$, S. Dallison ${ }^{16}$, A. de Roeck ${ }^{8}$, P. Dervan ${ }^{15}$, K. Desch ${ }^{25}$, B. Dienes ${ }^{30, h}$, M.S. Dixit ${ }^{7}$, M. Donkers ${ }^{6}$, J. Dubbert ${ }^{31}$, E. Duchovni ${ }^{24}$, G. Duckeck ${ }^{31}$, I.P. Duerdoth ${ }^{16}$, P.G. Estabrooks ${ }^{6}$, E. Etzion ${ }^{22}$, F. Fabbri ${ }^{2}$, M. Fanti ${ }^{2}$, L. Feld ${ }^{10}$, P. Ferrari ${ }^{12}$, F. Fiedler ${ }^{8}$,

I. Fleck ${ }^{10}$, M. Ford ${ }^{5}$, A. Frey ${ }^{8}$, A. Fürtjes ${ }^{8}$, D.I. Futyan ${ }^{16}$, P. Gagnon ${ }^{12}$, J.W. Gary ${ }^{4}$,

G. Gaycken ${ }^{25}$, C. Geich-Gimbel ${ }^{3}$, G. Giacomelli ${ }^{2}$, P. Giacomelli ${ }^{8}$, D. Glenzinski ${ }^{9}$,

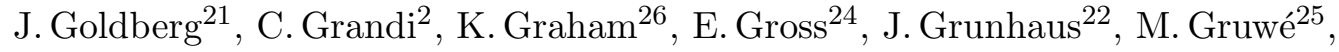
P.O. Günther ${ }^{3}$, C. Hajdu ${ }^{29}$, G.G. Hanson ${ }^{12}$, M. Hansroul ${ }^{8}$, M. Hapke ${ }^{13}$, K. Harder ${ }^{25}$, A. Harel ${ }^{21}$, C.K. Hargrove ${ }^{7}$, M. Harin-Dirac ${ }^{4}$, A. Hauke ${ }^{3}$, M. Hauschild ${ }^{8}$, C.M. Hawkes ${ }^{1}$, R.Hawkings ${ }^{25}$, R.J.Hemingway ${ }^{6}$, C. Hensel ${ }^{25}$, G. Herten ${ }^{10}$, R.D. Heuer ${ }^{25}$, M.D. Hildreth ${ }^{8}$, J.C. Hill ${ }^{5}$, A. Hocker ${ }^{9}$, K. Hoffman ${ }^{8}$, R.J.Homer ${ }^{1}$, A.K. Honma ${ }^{8}$, D. Horváth ${ }^{29, c}$,

K.R. Hossain ${ }^{28}$, R. Howard ${ }^{27}$, P. Hüntemeyer ${ }^{25}$, P. Igo-Kemenes ${ }^{11}$, K. Ishii ${ }^{23}$, F.R. Jacob ${ }^{20}$,

A. Jawahery ${ }^{17}$, H. Jeremie ${ }^{18}$, C.R. Jones ${ }^{5}$, P. Jovanovic ${ }^{1}$, T.R. Junk ${ }^{6}$, N. Kanaya ${ }^{23}$, J. Kanzaki ${ }^{23}$, G. Karapetian ${ }^{18}$, D. Karlen ${ }^{6}$, V. Kartvelishvili ${ }^{16}$, K. Kawagoe ${ }^{23}$, T. Kawamoto ${ }^{23}$, R.K. Keeler ${ }^{26}$, R.G. Kellogg ${ }^{17}$, B.W.Kennedy ${ }^{20}$, D.H. Kim ${ }^{19}$, K. Klein ${ }^{11}$, A. Klier ${ }^{24}$, T.Kobayashi ${ }^{23}$, M. Kobel ${ }^{3}$, T.P. Kokott ${ }^{3}$, S. Komamiya ${ }^{23}$, R.V.Kowalewski ${ }^{26}$, T. Kress ${ }^{4}$, P. Krieger ${ }^{6}$, J. von Krogh ${ }^{11}$, T. Kuhl ${ }^{3}$, M. Kupper ${ }^{24}$, P. Kyberd ${ }^{13}$, G.D. Lafferty ${ }^{16}$, H. Landsman ${ }^{21}$, D. Lanske ${ }^{14}$, I. Lawson ${ }^{26}$, J.G. Layter ${ }^{4}$, A. Leins ${ }^{31}$, D. Lellouch ${ }^{24}$, J. Letts ${ }^{12}$, L. Levinson ${ }^{24}$, R. Liebisch ${ }^{11}$, J. Lillich ${ }^{10}$, B. List ${ }^{8}$, C. Littlewood ${ }^{5}$, A.W. Lloyd ${ }^{1}$, S.L. Lloyd ${ }^{13}$, F.K. Loebinger ${ }^{16}$, G.D. Long ${ }^{26}$, M.J. Losty ${ }^{7}$, J. Lu $^{27}$, J. Ludwig ${ }^{10}$, A. Macchiolo ${ }^{18}$, A. Macpherson ${ }^{28}$, W. Mader ${ }^{3}$, M. Mannelli ${ }^{8}$, S. Marcellini' ${ }^{2}$, T.E. Marchant ${ }^{16}$, A.J. Martin ${ }^{13}$, J.P. Martin ${ }^{18}$, G. Martinez ${ }^{17}$, T. Mashimo ${ }^{23}$, P. Mättig ${ }^{24}$,

W.J. McDonald ${ }^{28}$, J. McKenna ${ }^{27}$, T.J.McMahon ${ }^{1}$, R.A. McPherson ${ }^{26}$, F. Meijers ${ }^{8}$,

P. Mendez-Lorenzo ${ }^{31}$, F.S. Merritt ${ }^{9}$, H. Mes ${ }^{7}$, A. Michelini' ${ }^{2}$, S. Mihara ${ }^{23}$, G. Mikenberg ${ }^{24}$, D.J. Miller ${ }^{15}$, W. Mohr ${ }^{10}$, A. Montanari ${ }^{2}$, T. Mori ${ }^{23}$, K. Nagai ${ }^{8}$, I. Nakamura ${ }^{23}$, H.A. Neal ${ }^{12, f}$, R. Nisius ${ }^{8}$, S.W. O'Neale ${ }^{1}$, F.G. Oakham ${ }^{7}$, F. Odorici ${ }^{2}$, H.O. Ogren ${ }^{12}$, A. $\mathrm{Oh}^{8}$, A. Okpara ${ }^{11}$, M.J. Oreglia ${ }^{9}$, S. Orito ${ }^{23}$, G. Pásztor ${ }^{8, j}$, J.R. Pater ${ }^{16}$, G.N. Patrick ${ }^{20}$, J. Patt ${ }^{10}$, P. Pfeifenschneider ${ }^{14}$, J.E. Pilcher ${ }^{9}$, J.Pinfold ${ }^{28}$, D.E. Plane ${ }^{8}$, B. Poli ${ }^{2}$, J. Polok ${ }^{8}$,

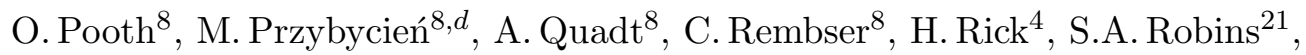

N. Rodning ${ }^{28}$, J.M. Roney ${ }^{26}$, S. Rosati ${ }^{3}$, K. Roscoe ${ }^{16}$, A.M. Rossi ${ }^{2}$, Y. Rozen ${ }^{21}$, K. Runge ${ }^{10}$, O. Runolfsson ${ }^{8}$, D.R. Rust ${ }^{12}$, K. Sachs ${ }^{6}$, T. Saeki ${ }^{23}$, O. Sahr ${ }^{31}$, E.K.G. Sarkisyan ${ }^{22}$, C. Sbarra ${ }^{26}$, A.D. Schaile ${ }^{31}$, O. Schaile ${ }^{31}$, P. Scharff-Hansen ${ }^{8}$, S. Schmitt ${ }^{11}$, M. Schröder ${ }^{8}$, M. Schumacher ${ }^{25}$, C. Schwick ${ }^{8}$, W.G. Scott ${ }^{20}$, R. Seuster ${ }^{14, h}$, T.G. Shears ${ }^{8}$, B.C.Shen ${ }^{4}$, C.H. Shepherd-Themistocleous ${ }^{5}$, P.Sherwood ${ }^{15}$, G.P. Siroli ${ }^{2}$, A.Skuja ${ }^{17}$, A.M. Smith ${ }^{8}$, G.A.Snow ${ }^{17}$, R. Sobie ${ }^{26}$, S.Söldner-Rembold ${ }^{10, e}$, S.Spagnolo ${ }^{20}$, M. Sproston ${ }^{20}$, A.Stahl ${ }^{3}$, K. Stephens ${ }^{16}$, K. Stoll ${ }^{10}$, D. Strom ${ }^{19}$, R. Ströhmer ${ }^{31}$, B. Surrow ${ }^{8}$, S.D. Talbot ${ }^{1}$, S. Tarem ${ }^{21}$, R.J. Taylor ${ }^{15}$, R. Teuscher ${ }^{9}$, M. Thiergen ${ }^{10}$, J. Thomas ${ }^{15}$, M.A. Thomson ${ }^{8}$, E. Torrence ${ }^{9}$, S. Towers ${ }^{6}$, T. Trefzger ${ }^{31}$, I. Trigger ${ }^{8}$, Z. Trócsányi ${ }^{30, g}$, E. Tsur ${ }^{22}$, M.F. Turner-Watson ${ }^{1}$, 


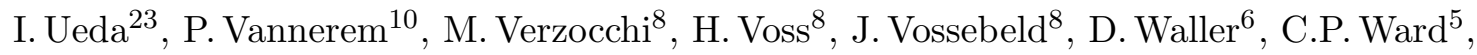
D.R. Ward ${ }^{5}$, P.M. Watkins ${ }^{1}$, A.T. Watson ${ }^{1}$, N.K. Watson ${ }^{1}$, P.S. Wells ${ }^{8}$, T. Wengler ${ }^{8}$, N. Wermes ${ }^{3}$, D. Wetterling ${ }^{11}$ J.S. White ${ }^{6}$, G.W.Wilson ${ }^{16}$, J.A. Wilson ${ }^{1}$, T.R. Wyatt ${ }^{16}$, S. Yamashita ${ }^{23}$, V. Zacek ${ }^{18}$, D. Zer-Zion ${ }^{8}$

${ }^{1}$ School of Physics and Astronomy, University of Birmingham, Birmingham B15 2TT, UK

${ }^{2}$ Dipartimento di Fisica dell' Università di Bologna and INFN, I-40126 Bologna, Italy ${ }^{3}$ Physikalisches Institut, Universität Bonn, D-53115 Bonn, Germany

${ }^{4}$ Department of Physics, University of California, Riverside CA 92521, USA

${ }^{5}$ Cavendish Laboratory, Cambridge CB3 0HE, UK

${ }^{6}$ Ottawa-Carleton Institute for Physics, Department of Physics, Carleton University, Ottawa, Ontario K1S 5B6, Canada

${ }^{7}$ Centre for Research in Particle Physics, Carleton University, Ottawa, Ontario K1S 5B6, Canada

${ }^{8}$ CERN, European Organisation for Nuclear Research, CH-1211 Geneva 23, Switzerland

${ }^{9}$ Enrico Fermi Institute and Department of Physics, University of Chicago, Chicago IL 60637, USA

${ }^{10}$ Fakultät für Physik, Albert Ludwigs Universität, D-79104 Freiburg, Germany

${ }^{11}$ Physikalisches Institut, Universität Heidelberg, D-69120 Heidelberg, Germany

${ }^{12}$ Indiana University, Department of Physics, Swain Hall West 117, Bloomington IN 47405, USA

${ }^{13}$ Queen Mary and Westfield College, University of London, London E1 4NS, UK

${ }^{14}$ Technische Hochschule Aachen, III Physikalisches Institut, Sommerfeldstrasse 26-28, D52056 Aachen, Germany

${ }^{15}$ University College London, London WC1E 6BT, UK

${ }^{16}$ Department of Physics, Schuster Laboratory, The University, Manchester M13 9PL, UK

${ }^{17}$ Department of Physics, University of Maryland, College Park, MD 20742, USA

${ }^{18}$ Laboratoire de Physique Nucléaire, Université de Montréal, Montréal, Quebec H3C 3J7, Canada

${ }^{19}$ University of Oregon, Department of Physics, Eugene OR 97403, USA

${ }^{20}$ CLRC Rutherford Appleton Laboratory, Chilton, Didcot, Oxfordshire OX11 0QX, UK

${ }^{21}$ Department of Physics, Technion-Israel Institute of Technology, Haifa 32000, Israel

${ }^{22}$ Department of Physics and Astronomy, Tel Aviv University, Tel Aviv 69978, Israel

${ }^{23}$ International Centre for Elementary Particle Physics and Department of Physics, University of Tokyo, Tokyo 113-0033, and Kobe University, Kobe 657-8501, Japan

${ }^{24}$ Particle Physics Department, Weizmann Institute of Science, Rehovot 76100, Israel

${ }^{25}$ Universität Hamburg/DESY, II Institut für Experimental Physik, Notkestrasse 85, D22607 Hamburg, Germany

${ }^{26}$ University of Victoria, Department of Physics, P O Box 3055, Victoria BC V8W 3P6, Canada

${ }^{27}$ University of British Columbia, Department of Physics, Vancouver BC V6T 1Z1, Canada

${ }^{28}$ University of Alberta, Department of Physics, Edmonton AB T6G 2J1, Canada

${ }^{29}$ Research Institute for Particle and Nuclear Physics, H-1525 Budapest, P O Box 49, Hungary

${ }^{30}$ Institute of Nuclear Research, H-4001 Debrecen, P O Box 51, Hungary 
${ }^{31}$ Ludwigs-Maximilians-Universität München, Sektion Physik, Am Coulombwall 1, D-85748 Garching, Germany

${ }^{a}$ and at TRIUMF, Vancouver, Canada V6T 2A3

${ }^{b}$ and Royal Society University Research Fellow

${ }^{c}$ and Institute of Nuclear Research, Debrecen, Hungary

$d$ and University of Mining and Metallurgy, Cracow

${ }^{e}$ and Heisenberg Fellow

${ }^{f}$ now at Yale University, Dept of Physics, New Haven, USA

$g$ and Department of Experimental Physics, Lajos Kossuth University, Debrecen, Hungary

$h$ and MPI München

${ }^{i}$ now at MPI für Physik, 80805 München

$j$ and Research Institute for Particle and Nuclear Physics, Budapest, Hungary.

\section{Introduction}

QCD predicts differences between quark and gluon jets. These are due to the different relative probabilities for a gluon and a quark to radiate an additional gluon, given by the $\mathrm{SU}(3)$ group constants $\mathrm{C}_{\mathrm{A}}=3$ and $\mathrm{C}_{\mathrm{F}}=4 / 3$. The various measurements of these differences from $\mathrm{e}^{+} \mathrm{e}^{-}$collider experiments are found to agree with the theoretical predictions: gluon jets are observed to have higher mean particle multiplicity, a softer fragmentation function and a wider angular spread than light quark jets [1]].

The QCD prediction of an enhancement of particle multiplicity in gluon jets with respect to quark jets is independent of the particle species, except for some small corrections. This prediction can be tested by measuring the rates of identified particles in gluon and quark jets. These measurements are also necessary for a better understanding of fragmentation processes and hadronisation models. The models presently used are mainly the string [2, 3] and cluster models [4 implemented in the Jetset [3] and Herwig [4] Monte Carlo generators. Both models are based on the parton shower approach, Jetset using a leading log perturbative QCD calculation, and Herwig a next-to-leading log calculation.

Some measurements of identified particle rates in quark and gluon jets have already been made by the LEP experiments [5], and the ratios of rates in quark and gluon jets have been determined. The ratios of rates of $\mathrm{K}^{+}, \mathrm{K}^{0}$, and protons were found to be consistent with the ratio of rates of the average charged particle multiplicity and also consistent with the ratio of rates determined from Monte Carlo simulations. The ratio of rates for $\Lambda$ was measured by OPAL [5] and found to be larger than the Monte Carlo expectation. The ratio of the $\eta$ meson production rate in 3-jet events to the $\eta$ production rate in 2-jet events was observed by the L3 experiment [6] to be larger than the Monte Carlo expectation. It was suggested that this difference could be caused by an enhanced $\eta$ meson production in gluon jets. A confirmation of this result would suggest that, in addition to the QCD predicted enhancement in gluon jets with respect to quark jets at equal jet energies, other $\eta$ meson sources might exist in gluon jets. Such sources could be production of glueballs and their decay to isoscalar mesons as has been foreseen in some theoretical models [0]. 
However, the ALEPH experiment [8] observed no evidence for an enhancement of $\eta$ mesons in gluon jets in excess of the Monte Carlo expectation.

Experimentally, results from the comparison of charged particle or identified particle rates in quark and gluon jets are highly dependent on event topologies (i.e. the jet localisation with respect to other jets in the event) and even more dependent on the jet energies. Furthermore, comparison between the experimental results and the QCD predictions is complicated by the use of jet-finding algorithms. Analytic calculations do not employ jet finders to assign particles to jets. To cope with these energy and topological dependences, a transverse momentum-like scale $\mathrm{Q}_{\text {jet }}$ (see Equation 2) has been proposed [9] and was used for a comparison of the mean charged particle multiplicity in quark and gluon jets [10].

In this paper, the rates of $\pi^{0}, \eta$ and $\mathrm{K}^{0}$ in quark and gluon jets are measured for the first time as a function of the scale $Q_{\text {jet }}$. A phenomenological formula for the charged particle rate $\mathrm{N}_{\mathrm{q}}\left(\mathrm{Q}_{\text {jet }}\right)$ in quark jets, written as a second order polynomial in $\ln \left(\mathrm{Q}_{\mathrm{jet}}\right)$, and a phenomenological formula for the charged particle rate $\mathrm{N}_{\mathrm{g}}\left(\mathrm{Q}_{\text {jet }}\right)$ in gluon jets, written as a linear function of $\mathrm{N}_{\mathrm{q}}\left(\mathrm{Q}_{\mathrm{jet}}\right)$, were used to describe simultaneously the observed charged particle multiplicities in quark and in gluon jets. The functions $\mathrm{N}_{\mathrm{q}}\left(Q_{\mathrm{jet}}\right)$ and $\mathrm{N}_{\mathrm{g}}\left(\mathrm{Q}_{\mathrm{jet}}\right)$, with all parameters set to the values obtained from a fit to the observed charged particle distributions, were used as a model to compare to the measured rates of identified particles in quark and gluon jets. For each comparison, only one overall normalisation factor, common to $\mathrm{N}_{\mathrm{q}}\left(\mathrm{Q}_{\text {jet }}\right)$ and $\mathrm{N}_{\mathrm{g}}\left(\mathrm{Q}_{\text {jet }}\right)$, is allowed to vary. This method provides a model independent way of testing that the enhancement of particle production in gluon jets with respect to quark jets is independent of the particle species.

\section{Data selection}

\subsection{Selection of hadronic $\mathrm{Z}^{0}$ decays}

The present analysis was based on the full hadronic event sample collected at centreof-mass energies at and near the $\mathrm{Z}^{0}$ peak by the OPAL detector from 1991 to 1995 . This corresponded to about 4 million hadronic $\mathrm{Z}^{0}$ decays. A full description of the OPAL detector can be found in [11]. Standard OPAL selection criteria were applied for track and electromagnetic cluster selection [12]. Tracks were required to have: at least 20 measured points in the jet chamber, a measured momentum greater than $0.10 \mathrm{GeV}$, an impact parameter $\left|\mathrm{d}_{0}\right|$ in the $\mathrm{r}-\phi$ plane smaller than $2 \mathrm{~cm}$, a $\mathrm{z}$ position within $25 \mathrm{~cm}$ of the interaction point and a measured angle with respect to the beam axis of greater than $20^{\circ}$. Electromagnetic clusters were required to have an energy greater than $0.1 \mathrm{GeV}$ if they were in the barrel part of the detector (i.e. $|\cos \theta| \leq 0.82)$ or greater than $0.3 \mathrm{GeV}$ if they were in the endcap part. The selected tracks and clusters not associated with tracks were fed, as four-vectors, to the jetfinding algorithms. Background from all sources was reduced to less than $0.8 \%$ and was neglected throughout the analysis. It was reduced by requiring for each event more than 7 measured tracks, a visible energy (i.e. the sum of detected particle energies after correcting for double counting) larger than $60 \mathrm{GeV}$ and an angle larger than $25^{\circ}$ between the calculated thrust axis and the beam axis . 


\subsection{Event simulation}

Detector effects and detection efficiencies for the studied particles were evaluated using 8 million Monte Carlo hadronic $\mathrm{Z}^{0}$ decays. Events were generated using the Jetset program tuned to reproduce the global features of hadronic events as measured with the OPAL detector. About 4 million events generated by the Herwig program were also used for comparison. The generated events were processed through a full simulation of the OPAL detector 13 and were processed using the same reconstruction and selection programs as were applied to the data.

\subsection{Selection of 3 -jet events}

Three jet-finding algorithms were used: Luclus [3], Durham [14] and the cone [15] jet finder. The Luclus jet finder was found to provide the best jet angular resolution, which was relevant for the present analysis. Luclus was therefore used as the reference algorithm while the two others were used for systematic comparisons. The jet algorithm was forced to resolve three jets in each hadronic event. The jet energies and momenta were then calculated by imposing energy and momentum conservation with planar massless kinematics [16], using the jet directions found by the jet algorithm. They are given by the cyclic relation:

$$
\mathrm{E}_{\mathrm{i}}=\frac{\sqrt{\mathrm{s}} \cdot \sin \theta_{\mathrm{j}, \mathrm{k}}}{\sin \theta_{\mathrm{i}, \mathrm{j}}+\sin \theta_{\mathrm{j}, \mathrm{k}}+\sin \theta_{\mathrm{k}, \mathrm{i}}},
$$

where $\theta_{\mathrm{i}, \mathrm{j}}$ is the angle between jet $\mathrm{i}$ and jet $\mathrm{j}$. The event was accepted as a 3 -jet event if each jet contained at least 3 charged particles, had a corrected energy exceeding $5 \mathrm{GeV}$, and pointed more than $20^{\circ}$ away from the beam axis and more than $30^{\circ}$ away from the direction of the other two jets.

The variable $\mathrm{Y}=\left(\mathrm{D}_{2 \rightarrow 3}-\mathrm{D}_{3 \rightarrow 4}\right) / \mathrm{E}_{\text {visible }}$ was used, where $\mathrm{D}_{2 \rightarrow 3}$ and $\mathrm{D}_{3 \rightarrow 4}$ were the Luclus jet algorithm resolution parameters $\left(D_{\text {join }}\right)$ [3] for the transition from 2 to 3 and 3 to 4 jets, respectively, and $E_{\text {visible }}$ the total visible energy. This variable measured the topological stability of the event as a 3 -jet event. For larger values of $Y$, the events tended to be three-fold symmetric, meaning that all inter-jet angles were close to $120^{\circ}$. To select stable 3 -jet events (i.e. events not close to the transition from three to four jets), only events with $\mathrm{Y}>0.2$ twere kept for further processing.

The total selected data sample contained approximately 493000 3-jet events, which was $12.32 \%$ of the total initial event sample. The corresponding fraction for Monte Carlo events was $12.30 \%$ for Jetset and $12.17 \%$ for Herwig.

For the Monte Carlo events, the jet-finding algorithm was applied at the parton, hadron and detector levels (defined in Section 3.2). At each level the jet energies were corrected to satisfy the constraints of energy and momentum conservation with planar massless kinematics, after which the jets were energy ordered, the first jet being the jet with the highest energy. The matching from parton to hadron level and from hadron to detector level was done using a simple angular correspondence: a jet at hadron level was matched with only one detector jet and one parton jet, those having the minimal angular deviation with respect to the hadron jet direction.

\footnotetext{
${ }^{1}$ The corresponding $\mathrm{Y}$ variable for Durham was defined as $\mathrm{Y}=\mathrm{y}_{2 \rightarrow 3}-\mathrm{y}_{3 \rightarrow 4}$, where $\mathrm{y}$ is the usual $\mathrm{y}_{\text {cut }}$ [14]. For the cone jet finder the inter-jet angles were considered instead.
} 
The jet energy resolution, defined as $\left(E_{\text {jet }}^{\text {parton }}-E_{\text {jet }}^{\text {detector }}\right) / E_{\text {jet }}^{\text {parton }}$, was found to range from $5 \%$ for the first jet to $13 \%$ for the third jet. The angle between the parton jet direction and the detector jet direction was found to have an r.m.s. of 0.07 radians for the highest energy jet and 0.16 radians for the lowest energy jet.

\section{Analysis method}

In this section, the jet scale $Q_{\text {jet }}$ and the jet purities are defined and the method of unfolding the average charged particle multiplicity to $100 \%$ pure quark and gluon jets is explained. In Section 4 the measured average charged particle multiplicity in pure quark and gluon jets, as a function of the scale $Q_{j e t}$, was fitted to phenomenological formulae. The purpose of the fit was to obtain an analytical shape that could be used as a reference or a model to compare in Section 6 to the corresponding shapes obtained in Section 5 for $\pi^{0}, \eta$ and $\mathrm{K}^{0}$. Systematic effects that could have distorted the measured shape are discussed in Section 4.3.

\subsection{Jet scale $Q_{\text {jet }}$}

It has already been shown [9] that the jet energy alone is not an adequate scale to describe the average particle multiplicity in quark and gluon jets. Coherence in QCD radiation suggests [17] that the position of a parton with respect to other partons in the event (i.e. the event topology) should also be considered in studies of jet properties. Inter-jet coherence effects that can lead to destructive or constructive interference effects on the particle flux in the inter-jet angular region, have been predicted and observed experimentally. An example is the string effect [18]. A transversemomentum-like scale combining the jet energy and its angular position with respect to the other jets has been used [17] in a phenomenological study of parton shower characteristics. This scale $Q_{j e t}$ was defined as:

$$
\mathrm{Q}_{\mathrm{jet}}=\mathrm{E}_{\mathrm{jet}} \sin (\theta / 2)
$$

where $\theta$ was the jet angle with respect to the closest jet. $Q_{\text {jet }}$ has already been used in an experimental study of multiplicity in $\mathrm{e}^{+} \mathrm{e}^{-} 3$-jet events [10]. The way the scale $\mathrm{Q}_{\text {jet }}$ incorporates the topological dependence can be seen, for example, at leading order in $\alpha_{\mathrm{s}}$ for the three parton $\mathrm{q} \overline{\mathrm{q}} \mathrm{g}$ vertex: colour being conserved in QCD, the gluon can be represented by a $q \bar{q}$ pair that compensates exactly the total colour charge of the initial q $\bar{q}$ pair. A mutual colour shielding occurs when the initial quark or anti-quark is close in angle to the gluon and reduces subsequent gluon radiation. This colour shielding is minimal for back-to-back partons, and the scale $Q_{\text {jet }}$ becomes equal to the parton energy for that case.

The scale $Q_{j e t}$ was used for the present analysis with no restriction on event topology except for the minimal inter-jet angle. The distributions of energy, $\mathrm{E}_{\mathrm{jet}}$, and scale, $Q_{\text {jet }}$ (normalised to the total number of analysed hadronic events), for the three energy ordered jets, are shown in Figures 1 $1 \mathrm{a}$ and $1 \mathrm{~b}$. The Monte Carlo distributions were found to reproduce the data very well. 


\subsection{Gluon jet definition and purity estimation}

The jet having the smallest energy, $E_{j e t}$, was found to have the highest gluon purity. The jet purities were estimated using the Monte Carlo information: the initial parton shower (parton level), the generated hadrons after the fragmentation processes (hadron level) and the reconstructed particles after the simulation of the full OPAL detector response (detector level). At the hadron level, all charged and neutral particles with lifetimes greater than $3 \times 10^{-10}$ s were considered as stable particles. A jet at the detector level was considered to be a gluon jet if it matched a parton jet that did not contain either the initial quark or anti-quark from the $\mathrm{Z}$ decay. The jet purities were determined directly from Monte Carlo as the fraction of quark or gluon jets present in the jet sample at a fixed scale $Q_{\text {jet }}$.

The purities could also be estimated from matrix element calculations. It has been shown [19] that, for leading order QCD matrix elements, the probability for a given jet $\{\mathrm{i}\}$ among the jets $\{\mathrm{i}, \mathrm{j}, \mathrm{k}\}$ to be a gluon jet can be expressed as a function of the jet energies:

$$
P_{i=g} \propto \frac{x_{j}^{2}+x_{k}^{2}}{\left(1-x_{j}\right)\left(1-x_{k}\right)},
$$

where $\mathrm{x}_{\mathrm{i}}=2 \mathrm{E}_{\mathrm{i}} / \sqrt{\mathrm{s}}$ and the corresponding probability for being a quark jet is

$$
\mathrm{P}_{\mathrm{i}=\mathrm{q}}=1-\mathrm{P}_{\mathrm{i}=\mathrm{g}},
$$

normalised to have:

$$
\mathrm{P}_{1=\mathrm{q}}+\mathrm{P}_{2=\mathrm{q}}+\mathrm{P}_{3=\mathrm{q}}=2 .
$$

The purities obtained using Monte Carlo information are shown in Figure 2 together with the purities obtained from the matrix elements. In the same figure are also shown the purities for OPAL data obtained from the matrix element formula. Very good agreement was obtained between the two methods, with less than $2 \%$ deviation in most of the range of $Q_{\text {jet }}$ common to the second and third jet (see Figure 1 and Figure 2). Therefore, the matrix element formula was used to estimate the jet purities directly from the data. The same agreement was also observed for Herwig Monte Carlo events.

\subsection{Unfolding to pure quark and gluon jets}

The measured jet samples were mixtures of quark and gluon jets, while meaningful comparison of gluon and quark jets should be performed on quantities evaluated for pure samples of gluon jets and pure samples of quark jets. This analysis used the average charged particle multiplicity for samples of jets at the same scale $Q_{\text {jet }}$ but having different gluon/quark jet purities. If $\left\langle\mathrm{N}_{1}\left(\mathrm{Q}_{\mathrm{jet}}\right)\right\rangle$ and $\left\langle\mathrm{N}_{2}\left(\mathrm{Q}_{\mathrm{jet}}\right)\right\rangle$ are the average measured charged particle multiplicities for two jet samples with different quark jet purities $\mathrm{P}_{1}\left(\mathrm{Q}_{\mathrm{jet}}\right)$ and $\mathrm{P}_{2}\left(\mathrm{Q}_{\mathrm{jet}}\right)$ at the same $\mathrm{Q}_{\mathrm{jet}}$ value, then:

$$
\begin{aligned}
& \left\langle\mathrm{N}_{1}\left(\mathrm{Q}_{\mathrm{jet}}\right)\right\rangle=\mathrm{P}_{1}\left(\mathrm{Q}_{\mathrm{jet}}\right)\left\langle\mathrm{N}_{\mathrm{q}}\left(\mathrm{Q}_{\mathrm{jet}}\right)\right\rangle+\left(1-\mathrm{P}_{1}\left(\mathrm{Q}_{\mathrm{jet}}\right)\right)\left\langle\mathrm{N}_{\mathrm{g}}\left(\mathrm{Q}_{\mathrm{jet}}\right)\right\rangle \\
& \left\langle\mathrm{N}_{2}\left(\mathrm{Q}_{\mathrm{jet}}\right)\right\rangle=\mathrm{P}_{2}\left(\mathrm{Q}_{\mathrm{jet}}\right)\left\langle\mathrm{N}_{\mathrm{q}}\left(\mathrm{Q}_{\mathrm{jet}}\right)\right\rangle+\left(1-\mathrm{P}_{2}\left(\mathrm{Q}_{\mathrm{jet}}\right)\right)\left\langle\mathrm{N}_{\mathrm{g}}\left(\mathrm{Q}_{\mathrm{jet}}\right)\right\rangle
\end{aligned}
$$


where $\left\langle\mathrm{N}_{\mathrm{q}}\left(\mathrm{Q}_{\text {jet }}\right)\right\rangle$ and $\left\langle\mathrm{N}_{\mathrm{g}}\left(\mathrm{Q}_{\text {jet }}\right)\right\rangle$ are the average particle multiplicities for $100 \%$ pure quark and gluon jet sample. This gives:

$$
\begin{gathered}
\mathrm{N}_{\mathrm{q}}\left(\mathrm{Q}_{\text {jet }}\right)=\frac{\left(1-\mathrm{P}_{2}\left(\mathrm{Q}_{\text {jet }}\right)\right)\left\langle\mathrm{N}_{1}\left(\mathrm{Q}_{\text {jet }}\right)\right\rangle+\left(1-\mathrm{P}_{1}\left(\mathrm{Q}_{\text {jet }}\right)\right)\left\langle\mathrm{N}_{2}\left(\mathrm{Q}_{\text {jet }}\right)\right\rangle}{\mathrm{P}_{1}\left(\mathrm{Q}_{\mathrm{jet}}\right)-\mathrm{P}_{2}\left(\mathrm{Q}_{\text {jet }}\right)} \\
\mathrm{N}_{\mathrm{g}}\left(\mathrm{Q}_{\text {jet }}\right)=\frac{\mathrm{P}_{1}\left(\mathrm{Q}_{\text {jet }}\right)\left\langle\mathrm{N}_{2}\left(\mathrm{Q}_{\text {jet }}\right)\right\rangle-\mathrm{P}_{2}\left(\mathrm{Q}_{\text {jet }}\right)\left\langle\mathrm{N}_{1}\left(\mathrm{Q}_{\text {jet }}\right)\right\rangle}{\mathrm{P}_{1}\left(\mathrm{Q}_{\text {jet }}\right)-\mathrm{P}_{2}\left(\mathrm{Q}_{\text {jet }}\right)} .
\end{gathered}
$$

This unfolding was only possible in the region where the $\mathrm{Q}_{\text {jet }}$ scales of the jet samples overlapped. The $\mathrm{Q}_{\text {jet }}$ distributions of the energy ordered jets (see Figure (1) showed that the second and the third jets could be considered to be different samples over a common range of $\mathrm{Q}_{\text {jet }}$ from 6 to $26 \mathrm{GeV}$.

\section{Average charged particle multiplicities}

\subsection{Measurements and parametrisation}

The average number of charged particles, $\left\langle\mathrm{N}^{\mathrm{ch}}\right\rangle$, per jet in bins of $1 \mathrm{GeV}$ of the scale $Q_{j e t}$ was measured for samples of the second and third jets, being respectively quark enriched and gluon enriched through jet energy ordering and having a wide common range of $Q_{\text {jet }}$. In each bin of $Q_{\text {jet }}$, the average purity was obtained from the matrix element formula. The efficiency corrections from detector to hadron level were calculated from Monte Carlo information as a function of $Q_{j e t}$ for each jet sample separately. The efficiency was defined as the ratio of the average number of charged particles at the detector level for a given jet sample at a given scale $Q_{j e t}$, divided by the equivalent quantity for the corresponding jet sample at the hadron level. The efficiencies were found to be approximately independent of $\mathrm{Q}_{\text {jet }}$ for all jet samples, and the corresponding corrections to $\left\langle\mathrm{N}^{\mathrm{ch}}\left(\mathrm{Q}_{\text {jet }}\right)\right\rangle$ were at the level of $10 \%$. The results obtained after unfolding to $100 \%$ pure quark and gluon jets are shown in Figure 3 . The unfolding was performed for $\mathrm{Q}_{\mathrm{jet}}>7 \mathrm{GeV}$, since the method worked for all three jet finders in this region.

The average charged particle multiplicities in quark jets, $\left\langle\mathrm{N}_{\mathrm{q}}\right\rangle$, and in gluon jets, $\left\langle\mathrm{N}_{\mathrm{g}}\right\rangle$, were simultaneously described with phenomenological formulae. The parametrisation was given by:

$$
\begin{gathered}
\left\langle\mathrm{N}_{\mathrm{q}}\left(\mathrm{Q}_{\mathrm{jet}}\right)\right\rangle=\mathrm{a}_{0}+\mathrm{a}_{1} \ln \mathrm{Q}_{\mathrm{jet}}+\mathrm{a}_{2}\left(\ln \mathrm{Q}_{\mathrm{jet}}\right)^{2} \\
\left\langle\mathrm{~N}_{\mathrm{g}}\left(\mathrm{Q}_{\mathrm{jet}}\right)\right\rangle=\mathrm{R}_{0}+\mathrm{R}_{1}\left\langle\mathrm{~N}_{\mathrm{q}}\left(\mathrm{Q}_{\mathrm{jet}}\right)\right\rangle
\end{gathered}
$$

where $\mathrm{a}_{0}, \mathrm{a}_{1}, \mathrm{a}_{2}, \mathrm{R}_{0}$ and $\mathrm{R}_{1}$ are constants. This parametrisation resembles the nextto-leading order expressions given in 20, 21], but with an extra offset parameter $R_{0}$. The leading order expressions [20, 21] alone were found to be unable to fit the data, as was also found in [10] and discussed in [22]. The $\mathrm{Q}_{\text {jet }}$ dependent average charged particle multiplicities for quark and gluon jets were fitted simultaneously to the expressions for $\left\langle\mathrm{N}_{1}\left(\mathrm{Q}_{\text {jet }}\right)\right\rangle$ and $\left\langle\mathrm{N}_{2}\left(\mathrm{Q}_{\text {jet }}\right)\right\rangle$ (Equations 6 and 7$)$ where $\left\langle\mathrm{N}_{\mathrm{q}}\left(\mathrm{Q}_{\text {jet }}\right)\right\rangle$ and $\left\langle\mathrm{N}_{\mathrm{q}}\left(\mathrm{Q}_{\text {jet }}\right)\right\rangle$ were replaced by the expressions given in equations 10 and 11 . The result of the fit yielded: $\mathrm{a}_{0}=2.74 \pm 0.07, \mathrm{a}_{1}=1.71 \pm 0.05, \mathrm{a}_{2}=-0.05 \pm 0.07, \mathrm{R}_{0}=-7.27 \pm 0.52$ and $\mathrm{R}_{1}=$ 
$2.27 \pm 0.07$. The fitted functions are shown with the data points unfolded using equations 8 and 9 in Figure 3 a, where a good description of the average charged particle multiplicities in both the gluon and the quark jets can be seen.

The ratio of the average charged particle multiplicities in gluon to quark jets is shown in Figure 3 $\mathrm{b}$. This ratio is predicted by QCD to be the same for all particle species. A test of this prediction is the quality of the fits of the above analytical function for charged particles to the measured multiplicities of $\pi^{0}, \eta$ and $\mathrm{K}^{0}$ in quark and gluon jets with all parameters fixed except for an overall normalisation factor.

\subsection{Ratio of the slopes of the multiplicities}

From QCD perturbative calculations, the ratio of particle multiplicities in gluon and quark jets is given by:

$$
\mathrm{N}_{\mathrm{g}}\left(\mathrm{Q}_{\mathrm{jet}}\right)=\mathrm{R}\left(\mathrm{Q}_{\mathrm{jet}}\right) \times \mathrm{N}_{\mathrm{q}}\left(\mathrm{Q}_{\text {jet }}\right)
$$

where the asymptotic limit of $\mathrm{R}\left(\mathrm{Q}_{\text {jet }}\right)$ at large $\mathrm{Q}_{\text {jet }}$ is an approximation to the $\mathrm{QCD}$ colour factor ratio $\mathrm{R}=\mathrm{C}_{\mathrm{A}} / \mathrm{C}_{\mathrm{F}}=2.25$. At the $\mathrm{Z}^{0}$ scale, this value is lower due to sizeable higher order corrections. However, the ratio of the slopes

$$
\mathrm{C}\left(\mathrm{Q}_{\text {jet }}\right)=\frac{\mathrm{d}\left\langle\mathrm{N}_{\mathrm{g}}\right\rangle / \mathrm{d} \mathrm{Q}_{\text {jet }}}{\mathrm{d}\left\langle\mathrm{N}_{\mathrm{q}}\right\rangle / \mathrm{d} Q_{\text {jet }}}\left(\mathrm{Q}_{\text {jet }}\right)
$$

of multiplicities in quark and gluon jets is expected to be less affected by these higher order corrections [23]. The slope ratio $\mathrm{C}$ has been recently calculated [23] using a nextto-next-to-next-to leading order (3NLO) perturbative approximation. The predicted value of $\mathrm{C}\left(\mathrm{Q}_{\text {jet }}\right)$ in $\mathrm{Z}^{0}$ decays is $\mathrm{C} \simeq 1.92$.

From the parametrisation given in equations 10 and 11 that imposes a constant value for $\mathrm{C}\left(\mathrm{Q}_{\text {jet }}\right)$ (Equation 13), we obtained:

$$
\mathrm{C}\left(\mathrm{Q}_{\mathrm{jet}}\right)=\mathrm{R}_{1}=2.27 \pm 0.07 \text { (stat.) } \pm 0.19 \text { (syst.). }
$$

The constraint $\mathrm{C}\left(\mathrm{Q}_{\text {jet }}\right)=$ constant can be released by extracting the spectrum $\mathrm{C}\left(\mathrm{Q}_{\text {jet }}\right)$ from the measured distributions $\left\langle\mathrm{N}_{\mathrm{q}}\left(\mathrm{Q}_{\mathrm{jet}}\right)\right\rangle$ and $\left\langle\mathrm{N}_{\mathrm{q}}\left(\mathrm{Q}_{\text {jet }}\right)\right\rangle$. This was done by using as an estimate of the derivative at each bin $Q_{j e t}$ the slope of a fitted line to three adjacent bins centred at $\mathrm{Q}_{\text {jet }}$. The obtained $\mathrm{C}\left(\mathrm{Q}_{\mathrm{jet}}\right)$ spectrum is shown in Figure $3 \mathrm{c}$, and a fit to a constant yielded:

$$
\mathrm{C}=2.27 \pm 0.09(\text { stat }) \pm 0.27 \text { (syst) }
$$

The systematic uncertainty, for both methods, was mainly due to differences between the jet finders and the correlations between the unfolded values of $\mathrm{N}_{\mathrm{g}}\left(Q_{\text {jet }}\right)$ and $\mathrm{N}_{\mathrm{q}}\left(\mathrm{Q}_{\mathrm{jet}}\right)$ at each bin of $\mathrm{Q}_{\text {jet }}$. The two values are about one standard deviation higher than the prediction [23] $\mathrm{C} \simeq 1.92$.

The Delphi collaboration recently presented a measurement of the ratio of slopes 10. Their result, $\mathrm{C}=1.97 \pm 0.10$ (stat), is about one standard deviation of the total uncertainty bellow our measurement. 


\subsection{Stability of the parametrisation of multiplicities}

To study systematic effects on the parametrisation of the charged particle multiplicity obtained in the previous section, several variations to the analysis procedure were applied. For each variation, new $\left\langle\mathrm{N}_{\mathrm{q}}\left(\mathrm{Q}_{\mathrm{jet}}\right)\right\rangle$ and $\left\langle\mathrm{N}_{\mathrm{q}}\left(\mathrm{Q}_{\mathrm{jet}}\right)\right\rangle$ were obtained and compared to the original distributions. At each data point, the deviation with respect to the original value is considered as a systematic error. The errors were added quadratically and were included in the error bars of the original data points shown in Figure 3. For each variation, the distributions $\left\langle\mathrm{N}_{\mathrm{q}}\left(\mathrm{Q}_{\mathrm{jet}}\right)\right\rangle$ and $\left\langle\mathrm{N}_{\mathrm{q}}\left(\mathrm{Q}_{\mathrm{jet}}\right)\right\rangle$ were also fitted to the functions of equations 10 and 11 . The resulting parametrisation was compared to the original one by calculating the largest relative difference between the new and the original function values. The following systematic variations were considered:

1. The analysis was completely repeated with the Durham and cone jet finders. The parametrisation was found to agree well between the Luclus (original) and the Durham jet finders. The parametrisation obtained using the cone jet finder agreed within $10 \%$ with the original one.

2. The analysis was repeated using a different method for estimating the efficiencies. The fully unfolded average charged particle multiplicities were obtained, as a function of $Q_{j e t}$ and for each jet sample, at the hadron level and then at the detector level. The ratio of detector to hadron level was then used to correct the average multiplicities of the data after having performed the unfolding. This procedure was done with the Jetset and Herwig Monte Carlos and the corrections were found to differ by at most 3\%. The analytic formula (Equations 10 and 11) was then fitted to the average charged particle multiplicity spectra corrected separately with Jetset and Herwig. The shape was found to agree with the original one within $2 \%$ for Jetset and $3 \%$ for Herwig.

3. The influence of the jet purities was studied by using the purities from the Monte Carlo matching, and also by increasing the cut on D from 0.20 to 0.25 . The effect on the fitted parametrisation was negligible.

4. Effects of soft particles on the measured multiplicities were studied by changing the minimum momentum required per track from $0.10 \mathrm{GeV}$ to $0.15 \mathrm{GeV}$, and the minimum number of tracks per jet was also changed from 3 to 5 . The analysis was repeated and the fitted parametrisation agreed with the original one within $3 \%$.

5. The analysis was repeated with two different jet samples. The first jet sample was gluon enriched by requiring that two jets were tagged as b-quark jets with a neural network b-tagging method and the remaining jet was considered as a gluon jet. The second jet sample, which was quark enriched, was obtained by selecting all second jets (energy ordered) in the events having no b-tagged quark jets. The average gluon purity was $80 \%$ for the first jet sample, and the average quark purity was $65 \%$ for the second jet sample. The fitted parametrisation agreed with the original one within $8 \%$. The new average charged particle multiplicity spectra, unfolded to $100 \%$ quark and gluon purities, agreed very well 
with the original spectra. Differences at each data point were considered as systematic errors; this should, in principle, also account for possible correlations between the measured $\mathrm{N}_{\mathrm{g}}\left(\mathrm{Q}_{\mathrm{jet}}\right)$ and $\mathrm{N}_{\mathrm{q}}\left(\mathrm{Q}_{\mathrm{jet}}\right)$.

\section{$5 \quad \pi^{0}, \eta$ and $\mathrm{K}^{0}$ meson production}

This section describes the reconstruction of the decay channels $\eta \rightarrow 2 \gamma, \pi^{0} \rightarrow 2 \gamma$ and $\mathrm{K}_{\mathrm{S}}^{0} \rightarrow \pi^{+} \pi^{-}$for the full $\mathrm{Z}^{0}$ hadronic decay sample without any 3 -jet requirement. In order to gain more confidence for the rate measurements in quark and gluon jets (see Section 6), the total inclusive rates as well as the differential rates in hadronic $\mathrm{Z}^{0}$ decays of $\pi^{0}, \eta$ and $\mathrm{K}^{0}$ were measured and compared to previous such measurements at LEP.

\section{$5.1 \quad \pi^{0}$ and $\eta$ reconstruction}

The reconstruction was restricted to the barrel region of the detector. A procedure [24] using a parametrisation of the expected lateral energy distribution of electromagnetic showers was optimised to resolve as many photon candidates as possible from the overlapping energy deposits in the electromagnetic calorimeter, in the dense environment of hadronic jets. The procedure was efficient but led to a rather low purity due to reconstructing spurious photons ("fakes"). Based on Monte Carlo simulated events, the rejection of fake photons was studied using a set of five measurable variables, namely: $\mathrm{E}_{\gamma}$ : the energy of the photon candidate, $\mathrm{E}_{\text {clust }}$ : the energy of the nearest cluster to the considered photon candidate, $\theta_{\text {clust }}$ : the opening angle between the photon candidate and the nearest cluster, $\theta_{\text {trk }}$ : the opening angle between the photon candidate and the closest reconstructed track, and $\mathrm{E}_{\text {trk }}$ : the amount of energy that could be attributed to tracks in an array of 3x3 lead glass blocks around the position of the photon candidate. A large number of obvious fake photons (mostly with $\mathrm{E}_{\gamma} \leq 300 \mathrm{MeV}$ ) were rejected with the following two cuts:

1. A candidate was rejected if it was found to satisfy:

$$
\mathrm{E}_{\gamma}<\mathrm{A} \cdot \mathrm{E}_{\text {clust }} \cdot \exp \left(-\left(\frac{\theta_{\text {clust }}}{40 \mathrm{mrad}}\right)^{2}\right) .
$$

Thus, when more than one photon candidate was obtained from a single electromagnetic cluster, candidates that had small energy compared to the cluster energy were rejected if their reconstructed position was close to the cluster centre. The $40 \mathrm{mrad}$ in the exponential is the average polar aperture of a lead glass block as seen from the interaction vertex. The factor A was determined empirically from the Monte Carlo sample. It was set at a value which ensured that the number of rejected photons was less than 1/10 of the number of rejected fake photons in all energy bins of $\mathrm{E}_{\gamma}$.

2. A candidate was also rejected if it was found to satisfy:

$$
\theta_{\text {trk }}<\mathrm{B}+\mathrm{C} \cdot \exp \left(-\left(\frac{\mathrm{E}_{\gamma}}{\mathrm{E}_{\mathrm{trk}}}\right)^{2}\right)
$$


A photon candidate was likely to be fake if it had an energy, $\mathrm{E}_{\gamma}$, smaller than the electromagnetic energy which could be attributed to tracks. The factors B and $\mathrm{C}$ were determined from the Monte Carlo sample. They were also set at values which ensured that the number of rejected photons was less than $1 / 10$ of the number of rejected fake photons in all energy bins of $\mathrm{E}_{\gamma}$.

The number of remaining fake photons was further reduced using a weight function

$$
\mathrm{W}\left(\mathrm{E}_{\gamma}, \mathrm{E}_{\text {clust }}, \theta_{\text {clust }}, \theta_{\text {trk }}, \mathrm{E}_{\text {trk }}\right)
$$

which was calculated for every photon candidate. The five variables were assumed uncorrelated and a likelihood ratio distribution was determined for each variable. The likelihood ratio in each bin was defined as the ratio of the number of generated photons to the total number of photon candidates. The value of $\mathrm{W}$ was the product of the five likelihood ratios of the bins $\mathrm{E}_{\gamma}, \mathrm{E}_{\text {clust }}, \theta_{\text {clust }}, \theta_{\text {trk }}$ and $\mathrm{E}_{\text {trk }}$ in which the candidate was found. The discriminating power of $\mathrm{W}$ is shown in Figure 1 , where the efficiency and purity for photons in Monte Carlo events are shown as a function of a value $\mathrm{W}_{\text {cut }}$. For photon candidates with $\mathrm{W}>\mathrm{W}_{\text {cut }}$, the purity was defined as the ratio of the number of generated photons to the total number of reconstructed photons and the efficiency was defined as the fraction of generated photons which were correctly reconstructed.

All possible pairs of photon candidates were then considered. Each pair was assigned a probability $\mathrm{P}$ for both candidates being correctly reconstructed photons, the probability being simply the product of the weights $\mathrm{W}$ associated to the two candidates:

$$
\mathrm{P}=\mathrm{W}_{1} \times \mathrm{W}_{2} \text {, with no cut on } \mathrm{W}_{1} \text { or } \mathrm{W}_{2} \text {. }
$$

The combinatorial background consisted of a mixture of three components: wrong pairing of two correctly reconstructed photons, pairing of two fake photons and pairing of one correctly reconstructed photon with one fake photon. Choosing only photon pairs with high values of $\mathrm{P}$ reduces the combinatorial background to its "wrong pairing of correctly reconstructed photons" component only. It was found that requiring $\mathrm{P}>0.1$ removed $60 \%$ of the total combinatorial background, with a relative loss in efficiency of $8 \%$ and $1 \%$ for $\pi^{0}$ and $\eta$ signals, respectively. The two-photon invariant mass distribution was studied in intervals of the energy fraction $\mathrm{x}_{\mathrm{E}}=\frac{\mathrm{E}_{2 \gamma}}{\mathrm{E}_{\mathrm{beam}}}$. Because most of the true reconstructed photons came from $\pi^{0}$ decays, an additional cut was required, in addition to the cut on the probability $\mathrm{P}$, to enhance the $\eta \rightarrow 2 \gamma$ signal. This cut excluded, for invariant masses $\mathrm{M}_{2 \gamma}>300 \mathrm{MeV}$, any photon that could pair with any other photon to make an invariant mass $\mathrm{M}_{2 \gamma}<300 \mathrm{MeV}$ with a probability $\mathrm{P}>0.1$.

The combinatorial background could be described by a second order polynomial for all cuts on probability $\mathrm{P}$, and for all $\mathrm{x}_{\mathrm{E}}$ bins. The signal was well described by a double Gaussian. The mass distributions obtained from the data over the full $\mathrm{x}_{\mathrm{E}}$ range are shown for four different cuts on the probability $\mathrm{P}$ in Figure 5 for $\pi^{0}$, and in Figure 6 for $\eta$. The absolute $\pi^{0}$ and $\eta$ reconstruction efficiencies, for $\mathrm{P}>0.1$ over the entire $\mathrm{x}_{\mathrm{E}}$ range, were $15.5 \%$ and $7 \%$ respectively. The efficiency and the signal to background ratio were dependent on the $\mathrm{x}_{\mathrm{E}}$ interval considered. 


\section{$5.2 \quad \mathrm{~K}_{\mathrm{S}}^{0}$ reconstruction}

The $\mathrm{K}_{\mathrm{S}}^{0} \rightarrow \pi^{+} \pi^{-}$reconstruction was similar to the method described in 25. Here it was applied to the full available $\mathrm{Z}^{0}$ hadronic sample of four million events. A track was considered to be a pion candidate if it had a transverse momentum larger than $150 \mathrm{MeV}$, had more than 20 hits in the jet chamber and had either more than 3 hits in the Z-chamber or a reconstructed end point in the jet chamber [26]. The invariant mass of pion pairs was evaluated for pairs of oppositely charged tracks having an intersection point in the plane perpendicular to the beam axis and satisfying the following requirements:

1. the distance from the intersection point to the primary vertex had to be greater than $1 \mathrm{~cm}$ and less than $150 \mathrm{~cm}$;

2. if the secondary vertex was reconstructed in the jet chamber, it had to be less than $5 \mathrm{~cm}$ from the first hit of either track;

3. if the intersection point occurred before the jet chamber, the radial distance from the track to the beam axis at the point of closest approach had to exceed $3 \mathrm{~mm}$;

4. track pairs that passed the above cuts were re-fitted with the constraint that they originated from a common vertex;

5. track pairs that satisfied the photon conversion hypothesis or $\Lambda \rightarrow p \pi$ hypothesis were rejected.

The $\pi^{+} \pi^{-}$invariant mass spectrum is shown in Figure 7 for the whole measured $\mathrm{x}_{\mathrm{E}}$ region. The spectrum was studied in $\mathrm{x}_{\mathrm{E}}$ intervals. In each interval, a double Gaussian shape for the signal and a second order polynomial for the background were used to fit the $\mathrm{M}_{\pi^{+} \pi^{-}}$spectrum. The $\mathrm{K}_{\mathrm{S}}^{0}$ reconstruction efficiency was found to be $26 \%$ for $\mathrm{x}_{\mathrm{E}}<0.1$, reducing to $15 \%$ at higher $\mathrm{x}_{\mathrm{E}}$.

\subsection{Inclusive $\pi^{0}, \eta$ and $\mathbf{K}^{0}$ rates}

The inclusive rate measurements were performed on the whole hadronic sample without making any 3-jet requirement. For each of the mesons $\pi^{0}, \eta$ and $\mathrm{K}_{\mathrm{S}}^{0}$, the number of entries remaining after the combinatorial background subtraction was considered as the number of signal entries. This was corrected for detector and reconstruction efficiencies and for the non-measured decay channels using the Particle Data Group branching ratios. The total rates were also corrected for the inaccessible $\mathrm{x}_{\mathrm{E}}$ regions. The corrections were performed using extrapolation with the spectral shapes predicted by Jetset. They amounted to $11 \%, 23 \%$ and $0.6 \%$ of the total rates for $\pi^{0}, \eta$ and $\mathrm{K}^{0}$ respectively. The $\mathrm{K}^{0}$ rate was the $\mathrm{K}_{\mathrm{S}}^{0}$ rate corrected for the non-observed $\mathrm{K}_{\mathrm{L}}^{0}$. The total inclusive measured rates per event were:

$$
\begin{gathered}
\left\langle\mathrm{n}_{\pi^{0}}\right\rangle=9.871 \pm 0.040 \text { (stat) } \pm 0.39 \text { (syst) } \\
\left\langle\mathrm{n}_{\eta}\right\rangle=1.076 \pm 0.090 \text { (stat) } \pm 0.084 \text { (syst) } \\
\left\langle\mathrm{n}_{\mathrm{K}^{0}}\right\rangle=2.016 \pm 0.003 \text { (stat) } \pm 0.052 \text { (syst) }
\end{gathered}
$$


The results are in good agreement with the values previously measured at LEP [25, 27]. The reconstruction method described in Section 5.1 gave an improved photon purity and higher $\pi^{0} \rightarrow 2 \gamma$ and $\eta \rightarrow 2 \gamma$ reconstruction efficiencies when compared to [24]. This led to a well controlled combinatorial background in the two-photon invariant mass spectrum. Thus, better systematic uncertainties were obtained. The statistical error on the $\eta$ inclusive rate was larger than the error quoted in [24] because the $\pi^{+} \pi^{-} \pi^{0}$ channel was not included in the present analysis. Since the full available $\mathrm{Z}^{0}$ hadronic sample was analysed, the statistical error on the $\mathrm{K}^{0}$ inclusive rate was improved when compared to the value quoted in [25]. However, the new systematic error was slightly larger, due to the inclusion of the systematic error on the Monte Carlo background and signal shapes (Section 5.4 item 1 to 3).

The rate measurement was repeated in $\mathrm{x}_{\mathrm{E}}$ intervals and the obtained differential rate distributions are shown in Figure 8. The $\pi^{0}$ measured differential rate was described well by both Jetset and Herwig Monte Carlo expectations. However, the measured $\eta$ and $\mathrm{K}^{0}$ spectra were harder than either Monte Carlo prediction. The discrepancy was worst for $\eta$, where the measured rate was almost double the predicted rate for $\mathrm{x}_{\mathrm{E}}>0.2$. The $\mathrm{K}^{0}$ measured rate was only about $15 \%$ larger than the predicted rate for $\mathrm{x}_{\mathrm{E}}>0.2$. For $\mathrm{x}_{\mathrm{E}}<0.1$ the Monte Carlo predicted rates for $\eta$ and $\mathrm{K}^{0}$ mesons agreed with the measured values within the error bars. These observations were in good agreement with previous OPAL results [24, 25]. The derived values for $\pi^{0}$ and $\eta$ are not meant to supersede the former OPAL results. The new measured $\mathrm{K}^{0}$ differential rates are given in Table 1.

\subsection{Systematic errors}

For the $\pi^{0}, \eta$ and $\mathrm{K}^{0}$ the largest contribution to the systematic error came from the parametrisation of the combinatorial background and the signal shape. This contributed up to $50 \%$ of the systematic error for some $\mathrm{x}_{\mathrm{E}}$ intervals. This error was estimated using the following procedure for each $\mathrm{x}_{\mathrm{E}}$ interval:

1. The shape of the background was measured from data by fitting a second order polynomial to the non-signal regions of the two-photon invariant mass spectrum. The procedure was repeated using different side bands.

2. The shape of the background was fixed to the shape predicted by the Monte Carlo and the background was fitted to data allowing only an overall normalisation factor to vary.

3. The shape of the signal was fixed to the shape predicted by the Monte Carlo and was fitted to data allowing only an overall normalisation factor to vary.

The systematic error was taken to be the quadratic sum of all deviations from the value measured using only data. For the $\mathrm{K}^{0}$ rate, the systematic errors in each $\mathrm{x}_{\mathrm{E}}$ interval were estimated using the procedure described in [25] to which was added the contribution from the three items above.

In the case of the $\eta$, the combinatorial background was found to have a small structure at $\mathrm{M}_{2 \gamma} \simeq 700 \mathrm{MeV}$ in the Monte Carlo which was not seen in the data. This structure was caused by $\omega(780) \rightarrow \pi^{0} \gamma \rightarrow 3 \gamma$ for which the rate in the OPALtuned Monte Carlo was twice the measured rate. To estimate the systematic error the above procedure was repeated by: 
1. fitting the background shape outside the signal and the small structure regions;

2. removing from the Monte Carlo $50 \%$ of the generated $\omega(780) \rightarrow 3 \gamma$, in which case the structure disappeared.

For both the $\pi^{0}$ and the $\eta$ all the following systematic variations were considered:

1. The systematic error relative to the cut on the probability $\mathrm{P}$ associated with each photon pair was estimated by repeating the measurements for each $\mathrm{x}_{\mathrm{E}}$ bin with different cuts on $\mathrm{P}>0.2, \mathrm{P}>0.3$ and $\mathrm{P}>0.4$, the original value being obtained with $\mathrm{P}>0.1$. This error, which took into account signal purity and reconstruction efficiency, since they depend on $\mathrm{P}$, was added quadratically to the previous error. In the worst case it contributed $14 \%$ to the total systematic error.

2. The difference between the correction factors for detector effects obtained with Jetset and those obtained with Herwig was considered as a systematic error. It was found to contribute up to $12 \%$ of the total systematic error.

3. The error due to the energy calibration of the electromagnetic calorimeter was estimated from Monte Carlo by shifting up and down the energy of measured electromagnetic clusters by $2 \%$. This had a negligible effect on the number of reconstructed photon candidates. However, the position of the peaks of the $\pi^{0}$ and $\eta$ signals were shifted by about $10 \mathrm{MeV}$, and the difference in the signal extracted in each $\mathrm{x}_{\mathrm{E}}$ bin was considered as a systematic error. In the worst case, this contributed $8 \%$ to the total systematic error.

4. Some of the mesons, mainly $\pi^{0}$, were produced in interactions with detector material. This effect might not have been very well modelled in the Monte Carlo. Therefore, half of the Monte Carlo prediction for these mesons was included in the uncertainty. This accounted for up to $25 \%$ of the total systematic error, the worst being in the low $\mathrm{x}_{\mathrm{E}}$ intervals.

5. An alternative extrapolation scheme as in [24] was used to correct for the inaccessible $\mathrm{x}_{\mathrm{E}}$ regions. This yielded slightly different corrections and these differences were included in the systematic error.

\section{$6 \quad \pi^{0}, \eta$ and $\mathbf{K}^{0}$ production in quark and gluon jets}

The $\pi^{0}, \eta$ and $\mathrm{K}^{0}$ yields in quark and gluon jets were estimated as a function of the scale $Q_{j e t}$. The average number of mesons produced in the second jet and third jet samples was measured as a function of $\mathrm{Q}_{\text {jet }}$, and then the unfolding to $100 \%$ quark and gluon jet purities was performed in the same way as was done for charged particles.

Each $\pi^{0}, \eta$ and $\mathrm{K}^{0}$ candidate was assigned to the jet which made the smallest opening angle with respect to the total momentum direction of the meson. The $\pi^{0}, \eta$ and $\mathrm{K}^{0}$ signals were then extracted as in the case of the inclusive rate measurement (see Section 5). To make the jets fully comparable, and because photons were reconstructed only in the barrel region of the electromagnetic calorimeter, only events that had both the second and third jet in the barrel were kept. The $Q_{\text {jet }}$ interval 
was divided into three bins: $Q_{\text {jet }}=7$ to 13,13 to 19 and 19 to $25 \mathrm{GeV}$. The average number of mesons per jet, in each $Q_{\text {jet }}$ interval, was corrected for detector acceptance, reconstruction efficiency and for inaccessible $\mathrm{x}_{\mathrm{E}}$ regions. Efficiencies were calculated separately for each jet as a function of $Q_{\text {jet }}$. Using the average purity of each bin of $Q_{\text {jet }}$ evaluated from data using the matrix element formula, the average number of mesons per jet was unfolded to $100 \%$ quark and gluon jet purities.

\section{1 $\pi^{0}$ production}

The average number of $\pi^{0}$ produced per jet as a function of $Q_{j e t}$ is shown in Figure 9 a for gluon and quark jets. The ratio of multiplicities in gluon and quark jets is shown in Figure 9b. The Jetset and Herwig models were found to reproduce the data within the error bars. This is demonstrated in Figure gc where the ratios of data to Monte Carlo are compatible with unity for both quark and gluon jets in each interval of $\mathrm{Q}_{\text {jet }}$. The analytical function obtained for the average charged particle multiplicity as a function of $\mathrm{Q}_{\text {jet }}$, scaled with only one free overall normalisation factor (measured to be 0.47 ), were found to fit well to the rate of $\pi^{0}$ as a function of $Q_{\text {jet }}$ in gluon and quark jets, and to the ratio between $\pi^{0}$ production rates in gluon and quark jets. The function is shown in Figures $9 \mathrm{a}$ and $9 \mathrm{~b}$.

\section{2 $\quad \eta$ production}

The same analytical function, but with an overall normalisation factor of 0.047 , was found to describe the $\eta$ rate in gluon and quark jets, as shown in Figure 10a. The ratio of $\eta$ multiplicities in gluon and quark jets as a function of $Q_{\text {jet }}$ is shown in Figure 10b, and was compatible with the ratio of charged particle multiplicities. The ratio was also compatible with being independent of $Q_{j e t}$, with

$$
\frac{\left\langle\mathrm{N}_{\mathrm{g}}^{\eta}\right\rangle}{\left\langle\mathrm{N}_{\mathrm{q}}^{\eta}\right\rangle}=1.29 \pm 0.14
$$

The measured $\eta$ rate was found to be slightly higher in the data than in the Monte Carlo, mainly at low $Q_{\text {jet }}$. This small disagreement was the same for both quark and gluon jets, as shown in Figure 10., where the ratios of data to Monte Carlo for gluon and quark jets are shown. This indicates that modelling of production of $\eta$ is equally inadequate for both Jetset and Herwig Monte Carlos. No additional enhancement of $\eta$ production in gluon jets was observed.

Figure 8 indicates that the measured $\eta$ spectrum was harder than the Monte Carlo prediction. To investigate this, the analysis was repeated for $\mathrm{x}_{\mathrm{E}} \geq 0.1$ for both $\pi^{0}$ and $\eta$ mesons. Due to statistical limitations, mainly in the $\eta$ meson sample, a yet harder cut on $\mathrm{x}_{\mathrm{E}}$ was not appropriate. The resulting $\pi^{0}$ production rates as a function of the scale $Q_{j e t}$ for the gluon and the quark jets were in good agreement with both Jetset and Herwig expectations. In each bin of $\mathrm{Q}_{\text {jet }}$, the ratio of data to Monte Carlo for the $\eta$ rate was equal for quark and gluon jets. The ratio of the $\eta$ production rates in data to those predicted by the Monte Carlo were found to be the same for both the quark and gluon jets. The ratio of the production rate of $\eta$ in gluon jets to that in quark 
jets was also found to be consistent with being independent of $\mathrm{Q}_{\mathrm{jet}}$. The measured ratio was:

$$
\frac{\left\langle\mathrm{N}_{\mathrm{g}}^{\eta}\left(\mathrm{x}_{\mathrm{E}}>0.1\right)\right\rangle}{\left\langle\mathrm{N}_{\mathrm{q}}^{\eta}\left(\mathrm{x}_{\mathrm{E}}>0.1\right)\right\rangle}=1.38 \pm 0.19 .
$$

This result is not in contradiction with previously published results [6] concerning an excess of high momentum $\eta$ mesons over Monte Carlo prediction. The excess is present equally in quark and gluon jets.

\section{3 $\quad \mathrm{K}^{0}$ production}

An overall scale factor of 0.093 in the analytical function of Section 4 gave the best fit to the $\mathrm{K}^{0}$ production rate in quark and gluon jets. However, as shown in Figure 11 a, the data for the quark jet were systematically but not significantly, higher than the corresponding analytical shape. This is also shown in Figure 11b where the ratio of $\mathrm{K}^{0}$ production in gluon and quark jets was systematically smaller than the corresponding ratio for charged particle production, although compatible within errors. This effect could be explained by a higher probability of producing a strange meson in the fragmentation of an initial strange, charmed or b quark.

The ratios data to Monte carlo of the $\mathrm{K}^{0}$ production rate in gluon and quark jets are shown in Figure 11c. The ratios are compatible with unity within errors and agree with the previous OPAL result [5].

\subsection{Systematic errors}

The measurements of the production rates of $\pi^{0}, \eta$ and $\mathrm{K}^{0}$ in jets were not statistically limited. The errors shown in Figures 9, 10 and 11 already include both the systematic and statistical errors added in quadrature and propagated through the unfolding formula. The systematic error ranged from $60 \%$ to $90 \%$ of the total quoted error, depending on the data point. It was estimated for each jet and each $\mathrm{Q}_{\text {jet }}$ interval using the procedure described in Section 6 for $\pi^{0}, \eta$ and $\mathrm{K}^{0}$ inclusive rate measurements. In each $Q_{j e t}$ interval, the selection criteria (see Section 5) for $\pi^{0}, \eta$ and $\mathrm{K}^{0}$ mesons were found to act equally on quark and gluon jets. The results were very stable against changes of the selection cuts that caused relative reconstruction efficiency variation of up to $5 \%$. For the $\eta$ case, the additional cut (Section 5.1) that excluded for invariant masses $\mathrm{M}_{2 \gamma}>300 \mathrm{MeV}$, any photon that could pair with any other photon to make an invariant mass $\mathrm{M}_{2 \gamma}<300 \mathrm{MeV}$ with a probability $\mathrm{P}>0.1$, was changed: first $\mathrm{M}_{2 \gamma}<300 \mathrm{MeV}$ was moved to $\mathrm{M}_{2 \gamma}<200 \mathrm{MeV}$ and second $\mathrm{P}>0.1$ was replaced by $\mathrm{P}>0.2$. The effect on the $\eta$ measured rates in quark and gluon jets was negligible $(<1 \%)$. In addition, for all studied mesons:

1. The difference between the measurements with purities taken from the matrix element formula and from Monte Carlo(see Section 3) was negligible. The average jet topologies and purities were varied by changing the cut on the variable Y (see Section 2.3) such that the purities obtained with the matrix element formula still agreed with those obtained from Monte Carlo information. The analysis was repeated with $\mathrm{Y} \leq 0.25$ and the differences obtained in each bin of $\mathrm{Q}_{\text {jet }}$ were considered as systematic errors and were found to contribute at most $5 \%$ of the total quadratic sum. 
2. Since the mesons were reconstructed independently of the jet-finding, and were assigned to jets by angular matching once the jets were reconstructed, very little dependence on the jet finder was expected. Indeed, this was the case for Luclus and Durham jet finders where the difference was measured to be less than $2 \%$. However, the results obtained with the cone jet finder showed deviations of up to $10 \%$ with respect to the two other jet finders. This was considered as a systematic error, and contributed up to $47 \%$ of the total quadratic sum of the systematic errors.

3. The results were found to be stable in each bin of $Q_{\text {jet }}$ with respect to changes of the charged particle selection requirements. The results were also found to be stable when the number of charged particles required per jet was increased from 3 to 5 . Lowering the cut on the inter-jet angle from 30 to $20^{\circ}$ was correlated to the cut on the variable $\mathrm{Y}$. The analysis was repeated with the minimum interjet angle set to $20^{\circ}$ and the minimum value of $\mathrm{Y}$ set to 0.22 . The maximum deviation obtained was less than $2 \%$ and was added to the total systematic error.

4. Due to statistical limitations, the analysis could not be repeated on data using b-tagging to obtain gluon enriched jet samples. A systematic error, that could account for correlations between particle content in pure quark and pure gluon jets as well as for the stability of the unfolding method, was assigned using the following procedure: The analysis was repeated on four different Jetset Monte Carlo samples, each sample being as large as the full available data sample. The jet samples were selected to have different gluon and quark average purities. The purities were set based on Monte Carlo information (Section 3.2) to be: (40\% quark, $60 \%$ gluon), (25\% quark, $75 \%$ gluon), ( $60 \%$ quark, $40 \%$ gluon), ( $75 \%$ quark, $25 \%$ gluon) for the four pairs of jet samples that were processed with the unfolding method. The largest deviation with respect to the average unfolded meson rate was considered as a systematic error. This contributed up to $7 \%$ of the systematic error, indicating that the unfolding procedure was indeed stable against large purity variations.

\section{$7 \quad$ Summary and conclusion}

Average multiplicities of $\pi^{0}, \eta, \mathrm{K}^{0}$ and charged particles have been measured for gluon and quark jets as a function of a transverse momentum-like scale $Q_{j e t}$. The average multiplicities were unfolded to $100 \%$ purity by comparing two jet samples having different gluon (or quark) content for the same value of $\mathrm{Q}_{\text {jet }}$. The $\pi^{0}, \eta$ and $\mathrm{K}^{0}$ inclusive production rates in $\mathrm{Z}^{0}$ hadronic decays were measured and found to agree with the previous values measured at LEP. The $\pi^{0}$ production rate was found to be well described by both Jetset and Herwig Monte Carlos for all values of $\mathrm{x}_{\mathrm{E}}$. The $\eta$ and $\mathrm{K}^{0}$ spectra were found to be harder than the Monte Carlo predictions, particularly in the case of $\eta$.

The charged particle multiplicity as a function of the topological scale $Q_{\text {jet }}$ for pure quark and pure gluon jets was described by a simple phenomenological formula. The same formula, with all parameters fixed except for an overall normalisation factor, 
was found to provide a good fit to the $\pi^{0}, \eta$ and $\mathrm{K}^{0}$ production rates in gluon and quark jets. The analysis showed that for $\pi^{0}, \eta$ and $\mathrm{K}^{0}$ mesons, there was no evidence for an enhancement in gluon jets with respect to quark jets beyond the enhancement observed for inclusive charged particles. In particular we observed no evidence for an enhancement of $\eta$ meson production in gluon jets, contrary to the predictions of some models for gluon jet hadronisation.

We measured the ratio of the slope of the average charged particle multiplicity in gluon jets to that in quark jets. We obtained $\mathrm{C}=2.227 \pm 0.07$ (stat.) \pm 0.19 (syst.) for this ratio, that is about one standard deviation of the total uncertainty above the analytic next-to-next-to-next-to leading order (3NLO) prediction 23.

\section{Acknowledgements}

We particularly wish to thank the SL Division for the efficient operation of the LEP accelerator at all energies and for their continuing close cooperation with our experimental group. We thank our colleagues from CEA, DAPNIA/SPP, CE-Saclay for their efforts over the years on the time-of-flight and trigger systems which we continue to use. In addition to the support staff at our own institutions we are pleased to acknowledge the

Department of Energy, USA,

National Science Foundation, USA,

Particle Physics and Astronomy Research Council, UK,

Natural Sciences and Engineering Research Council, Canada,

Israel Science Foundation, administered by the Israel Academy of Science and Humanities, Minerva Gesellschaft, Benoziyo Center for High Energy Physics, Japanese Ministry of Education, Science and Culture (the Monbusho) and a grant under the Monbusho International Science Research Program, Japanese Society for the Promotion of Science (JSPS),

German Israeli Bi-national Science Foundation (GIF),

Bundesministerium für Bildung und Forschung, Germany,

National Research Council of Canada,

Research Corporation, USA,

Hungarian Foundation for Scientific Research, OTKA T-029328, T023793 and OTKA F-023259.

\section{References}

[1] OPAL Collaboration, P.D. Acton et al., Z. Phys. C58 (1993) 387;

OPAL Collaboration, R. Akers et al., Z. Phys. C68 (1995) 179;

DELPHI Collaboration, P. Abreu et al., Z. Phys. C70 (1996) 179;

ALEPH Collaboration, D. Buskulic et al., Phys. Lett. B384 (1996) 353;

OPAL Collaboration, K. Ackerstaff et al., Phys. Lett. B388 (1996) 659; 
OPAL Collaboration, K. Ackerstaff et al., Eur. Phys. J. C1 (1998) 479;

OPAL Collaboration, K. Ackerstaff et al., Eur. Phys. J. C11 (1999) 217.

[2] I.G. Knowles, Nucl. Phys. B310 (1988) 571.

[3] T. Sjöstrand and M. Bengtsson, Comp. Phys. Comm. 43 (1987) 367.

[4] G. Marchesini and B. Webber, Nucl. Phys. B310 (1988) 461;

G. Marchesini et al., Comp. Phys. Comm. 67 (1992) 465.

[5] OPAL Collaboration, K. Ackerstaff et al., Eur. Phys. J. C8 (1999) 241;

L3 Collaboration, M. Acciari et al., Phys. Lett. B407 (1997) 389;

DELPHI Collaboration, P. Abreu et al., Phys. Lett. B401 (1997) 118.

[6] L3 Collaboration, M. Acciari et al., Phys. Lett. B371 (1996) 126.

[7] C. Peterson and T.F. Walsh, Phys. Lett. B91 (1980) 455.

[8] ALEPH Collaboration, D. Barate et al., CERN-EP/99-105.

[9] ALEPH Collaboration, D. Buskulic et al., Z. Phys. C76 (1997) 191.

[10] DELPHI Collaboration, P. Abreu et al., Phys. Lett. B449 (1999) 383.

[11] OPAL Collaboration, K. Ahmet et al., Nucl. Instr. and Meth. A305 (1991) 255;

P. P. Allport et al., Nucl. Instr. and Meth. A324 (1993) 34;

P. P. Allport et al., Nucl. Instr. and Meth. A346 (1994) 476.

[12] OPAL Collaboration, K. Ackerstaff et al., Phys. Lett. B388 (1996) 659.

[13] J. Allison et al., Nucl. Instr. and Meth. A317 (1992) 47.

[14] S. Catani et al., Phys. Lett. B269 (1991) 432.

[15] UA1 Collaboration, G. Arnison et al., Phys. Lett. B122 (1983) 103;

J.E. Huth et al., Snowmass(1990), Ed. E.L. Berger, World Scientific, Singapore (1990) 134;

OPAL Collaboration, R. Akers et al., Z. Phys. C63 (1994) 197.

[16] OPAL Collaboration, M.Z. Akrawy et al., Phys. Lett. B263 (1991) 311;

OPAL Collaboration, G. Alexander et al., Phys. Lett. B265 (1991) 462.

[17] Yu. Dokshitzer et al., Basics of perturbative QCD, Editions Frontières (1991).

[18] JADE Collaboration, W. Bartel et al., Phys. Lett. B101 (1981) 129;

JADE Collaboration, W. Bartel et al., Z. Phys. C21 (1983) 37;

TPC Collaboration, H. Aihara et al., Z. Phys. C28 (1985) 31;

OPAL Collaboration, G. Alexander et al., Phys. Lett. B261 (1991) 334;

OPAL Collaboration. R. Akers et al., Z. Phys. C68 (1995) 531.

[19] R.K. Ellis, D.A. Ross and A.E. Terrano, Nucl. Phys. B178 (1981) 421;

Z. Fodor, Phys Lett. B263 (1991) 306. 
[20] A.H. Müller, Nucl. Phys. B241, (1984) 141;

J.B. Gaffney and A.H. Müller, Nucl. Phys. B250 (1985) 109.

[21] B.R. Webber, Phys. Lett. B143 (1984) 501.

[22] J.W. Gary, hep-ex/9911011, UCRHEP-E265; Phys. Rev. D61 (2000) 114007.

[23] A. Capella et al., Phys. Rev. D61 (2000) 074009.

[24] OPAL Collaboration, K. Ackerstaff et al., Eur. Phys. J. C5 (1998) 411.

[25] OPAL Collaboration, R. Akers et al., Z. Phys. C67 (1995) 389;

OPAL Collaboration, G. Alexander et al., Phys. Lett. B264 (1991) 467.

[26] O. Biebel et. al., Nucl. Inst. and Meth. A323 (1992) 169.

[27] L3 Collaboration, M. Acciari et al., Phys. Lett. B259 (1991) 199;

ALEPH Collaboration, D. Buskulic et al., Z. Phys. C69 (1996) 379;

DELPHI Collaboration, P. Abreu et al., Z. Phys. C69 (1996) 561;

L3 Collaboration, M. Acciari et al., Phys. Lett. B371 (1996) 126;

ALEPH Collaboration, D. Buskulic et al., Z. Phys. C74 (1997) 451;

ALEPH Collaboration, D. Buskulic et al., Eur. Phys. J. C5 (1998) 205;

OPAL Collaboration, K. Ackerstaff et al., Eur. Phys. J. C5 (1998) 411. 


\begin{tabular}{|c|c|}
\hline $\mathrm{x}_{\mathrm{E}}$ range & $\frac{1}{\sigma_{\mathrm{had}}} \times \frac{\mathrm{d} \sigma}{\mathrm{dx}}$ \\
\hline \hline $0.0114-0.020$ & $25.731 \pm 0.232 \pm 1.430$ \\
$0.020-0.030$ & $24.617 \pm 0.120 \pm 1.300$ \\
$0.030-0.040$ & $19.349 \pm 0.116 \pm 1.040$ \\
$0.040-0.050$ & $15.500 \pm 0.061 \pm 0.767$ \\
$0.050-0.060$ & $13.170 \pm 0.072 \pm 0.690$ \\
$0.060-0.070$ & $11.144 \pm 0.073 \pm 0.600$ \\
$0.070-0.080$ & $9.360 \pm 0.066 \pm 0.500$ \\
$0.080-0.900$ & $8.470 \pm 0.061 \pm 0.468$ \\
$0.090-0.100$ & $7.010 \pm 0.059 \pm 0.401$ \\
$0.100-0.125$ & $5.734 \pm 0.029 \pm 0.312$ \\
$0.125-0.150$ & $4.488 \pm 0.028 \pm 0.247$ \\
$0.150-0.200$ & $3.100 \pm 0.019 \pm 0.169$ \\
$0.200-0.250$ & $1.945 \pm 0.015 \pm 0.104$ \\
$0.250-0.300$ & $1.266 \pm 0.010 \pm 0.071$ \\
$0.300-0.350$ & $0.860 \pm 0.010 \pm 0.050$ \\
$0.350-0.400$ & $0.579 \pm 0.009 \pm 0.035$ \\
$0.400-0.450$ & $0.394 \pm 0.008 \pm 0.026$ \\
$0.450-0.500$ & $0.253 \pm 0.005 \pm 0.018$ \\
$0.500-0.600$ & $0.163 \pm 0.003 \pm 0.018$ \\
$0.600-0.800$ & $0.051 \pm 0.001 \pm 0.010$ \\
\hline
\end{tabular}

Table 1: $\mathrm{K}^{0}$ differential rate. 

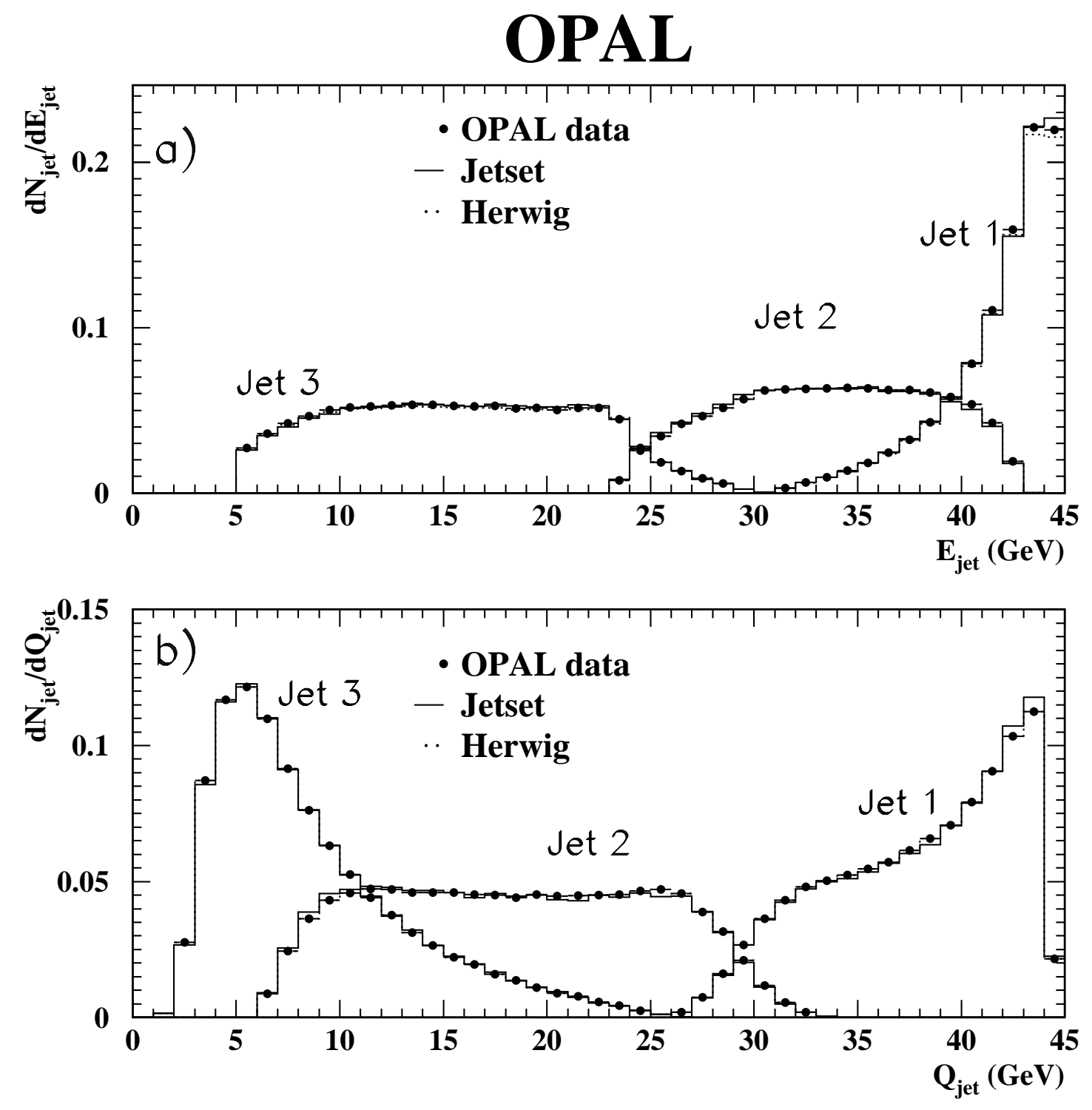

Figure 1: a) Jet energy $\left(E_{\text {jet } 1}>E_{\text {jet } 2}>E_{\text {jet } 3}\right)$ and $\left.b\right)$ jet scale $Q_{\text {jet }}=E_{\text {jet }} \sin (\theta / 2)$ distributions for the selected events. 


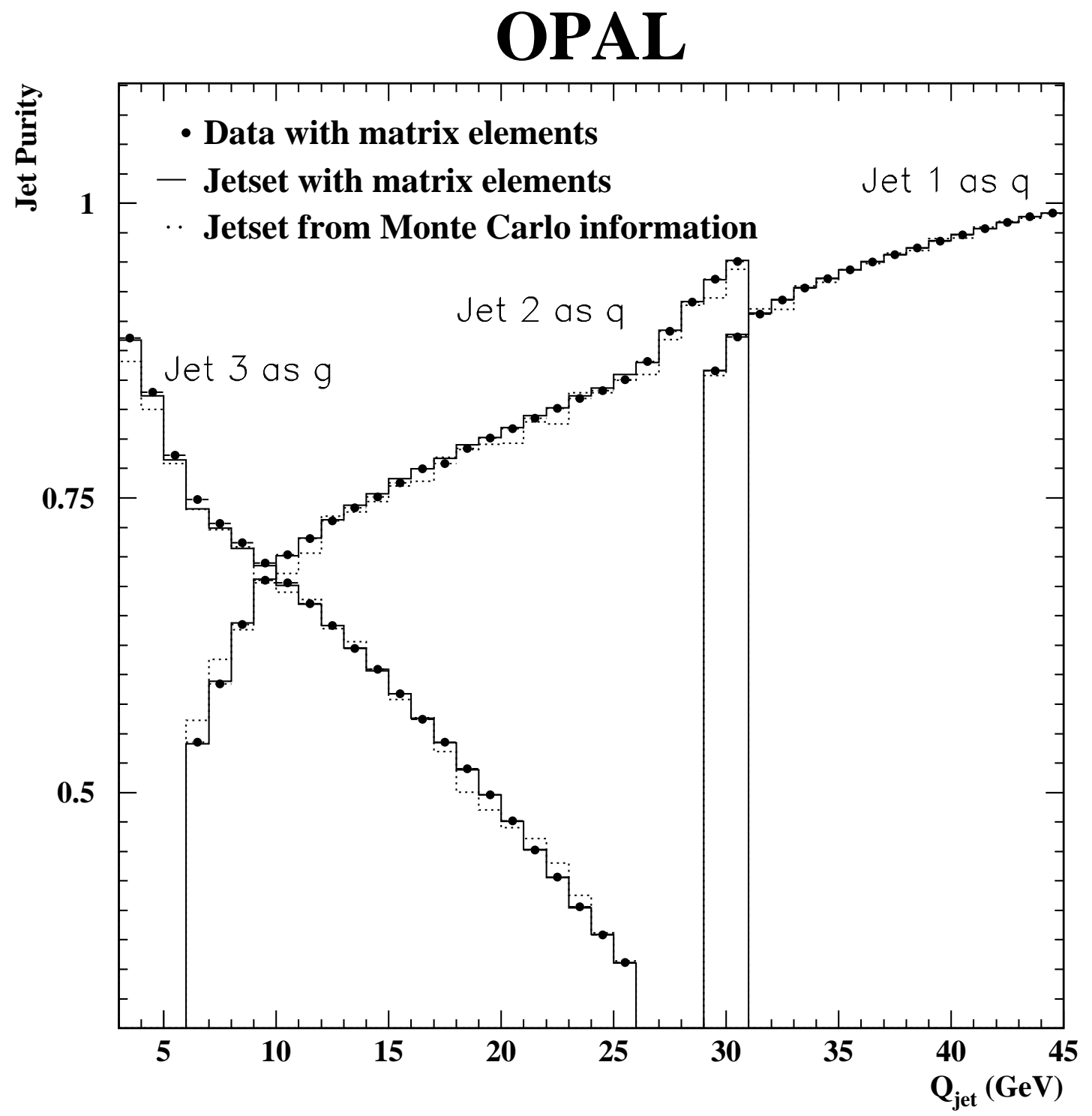

Figure 2: Quark jet purities of the first and second jet, and gluon jet purity of the third jet as a function of the scale $Q_{\text {jet }}$. Jets are energy ordered. The purities of OPAL data obtained from the matrix element formula are shown together with the purities for Jetset Monte Carlo events estimated from Monte Carlo information and from the matrix element formula. 


\section{OPAL}
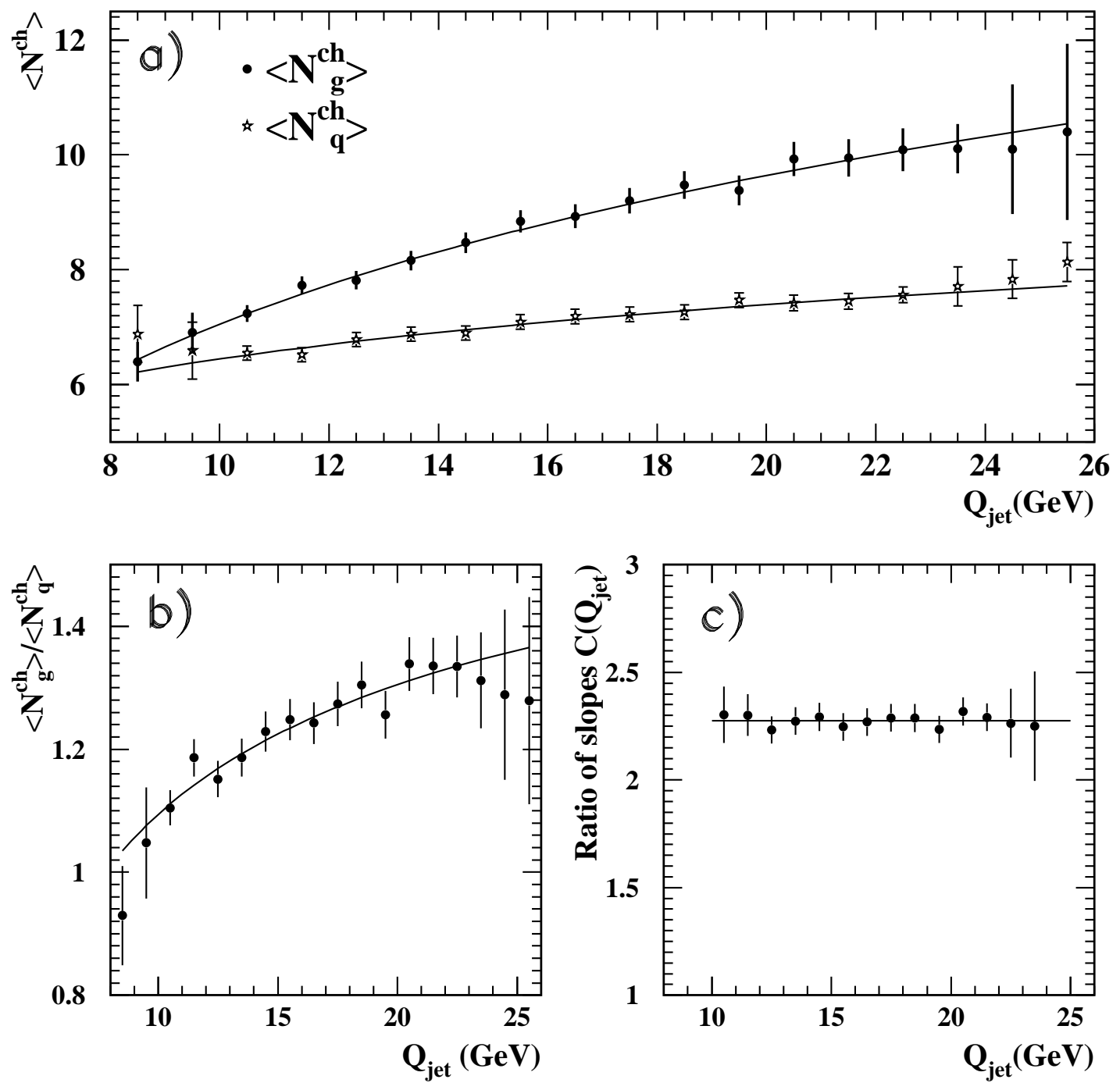

Figure 3: a) Average number of charged particles for pure gluon and quark jets as a function of the scale $Q_{\text {jet }}$. Systematic errors are included. The continuous curves are from a fit to a phenomenological formula (see text). b) Ratio of the average charged particle multiplicities in gluon and quark jets as a function of the scale $Q_{\text {jet }}$. c) Ratio of the average charged particle multiplicity slopes in gluon and quark jets; the line is a fit to a constant. 


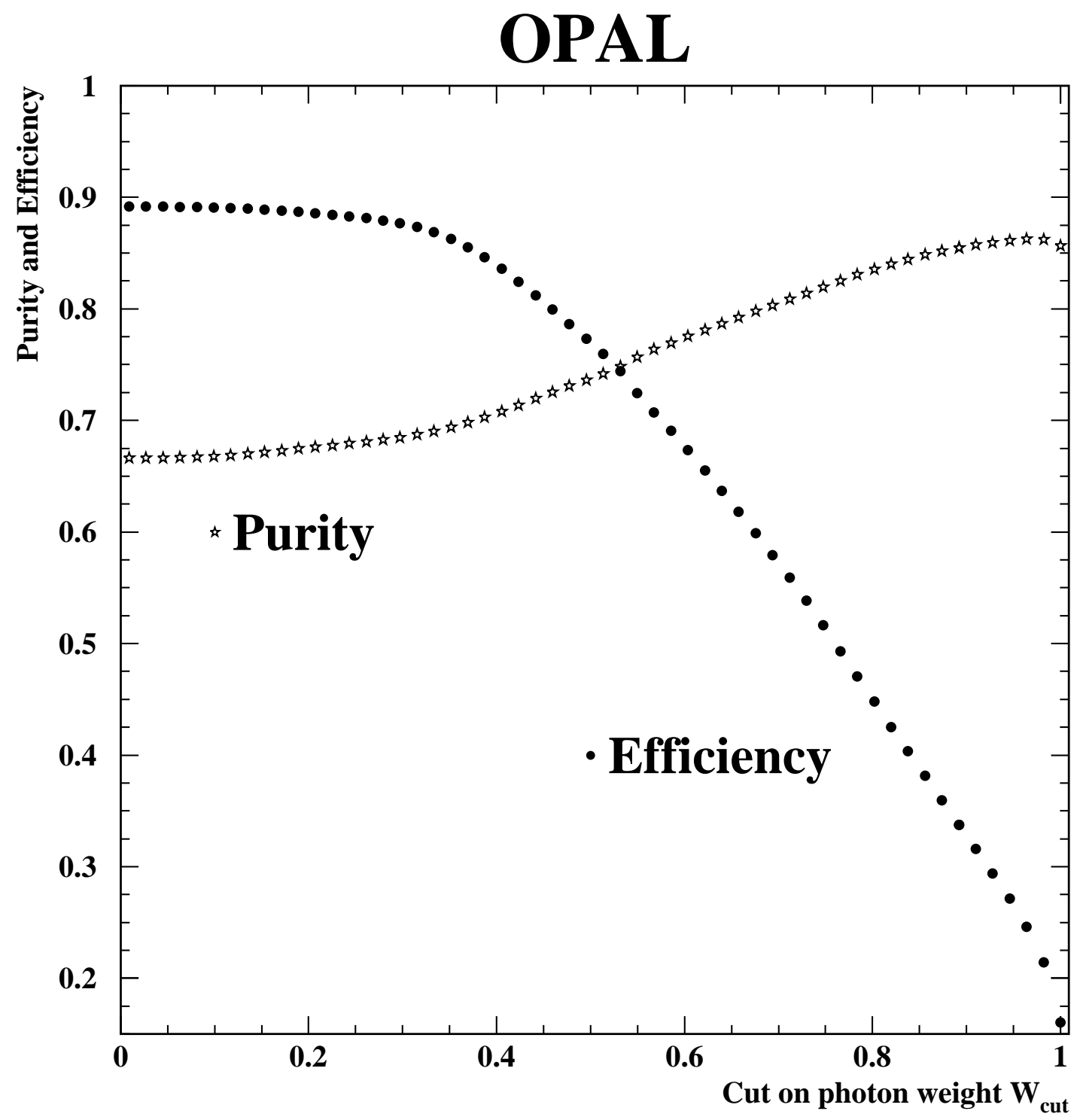

Figure 4: Purity and efficiency of the photon reconstruction versus the cut value on the associated weight $\mathrm{W}$. 
OPAL

$\times 1^{2}$
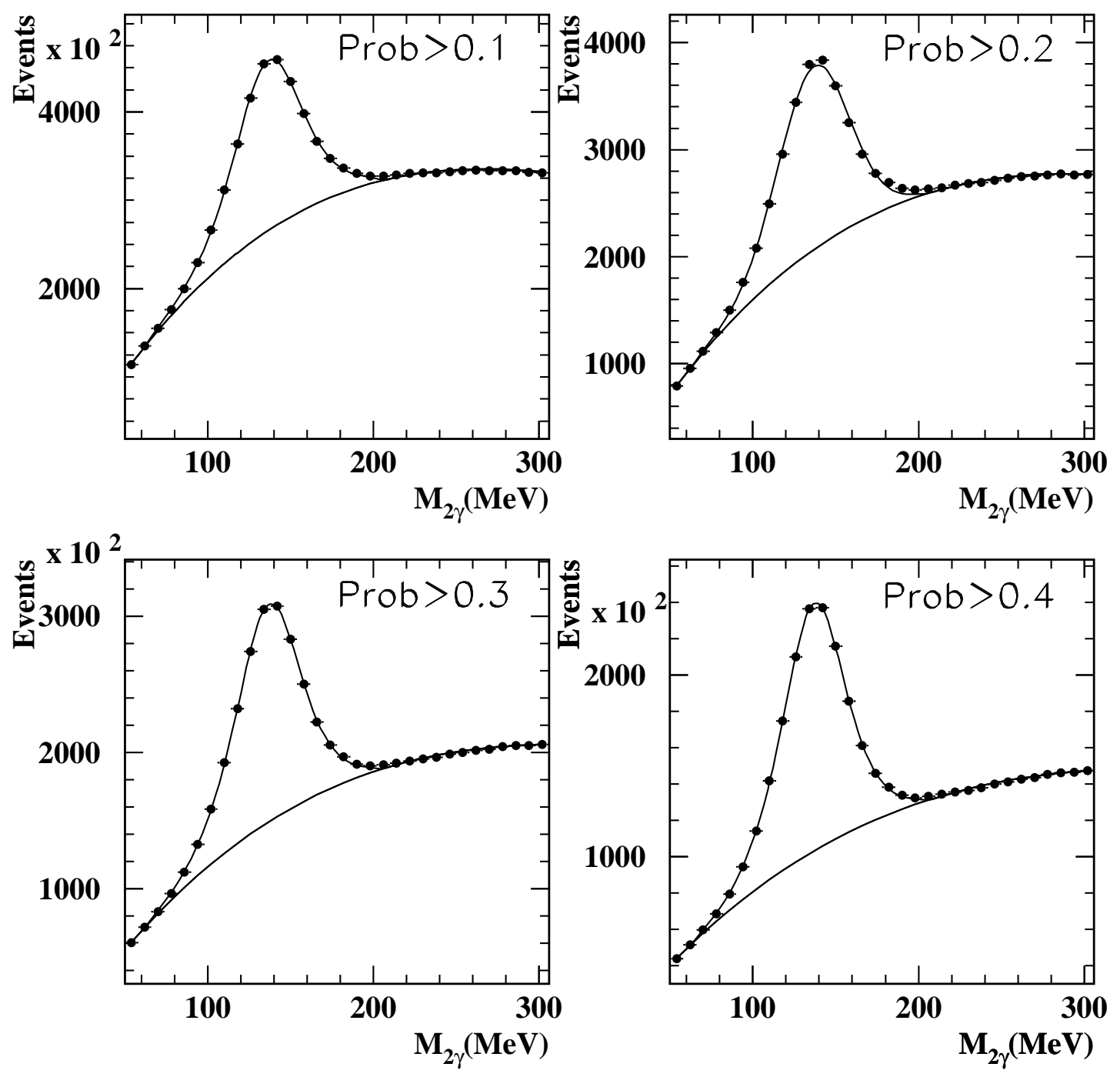

Figure 5: Two-photon invariant mass distribution in the vicinity of the $\pi^{0}$ for different cuts on the probability (prob) $\mathrm{P}=\mathrm{W}_{1} \times \mathrm{W}_{2}$. The smooth curve is from the fit to a double Gaussian and a second order polynomial. Note the suppressed zero on the vertical axis. 


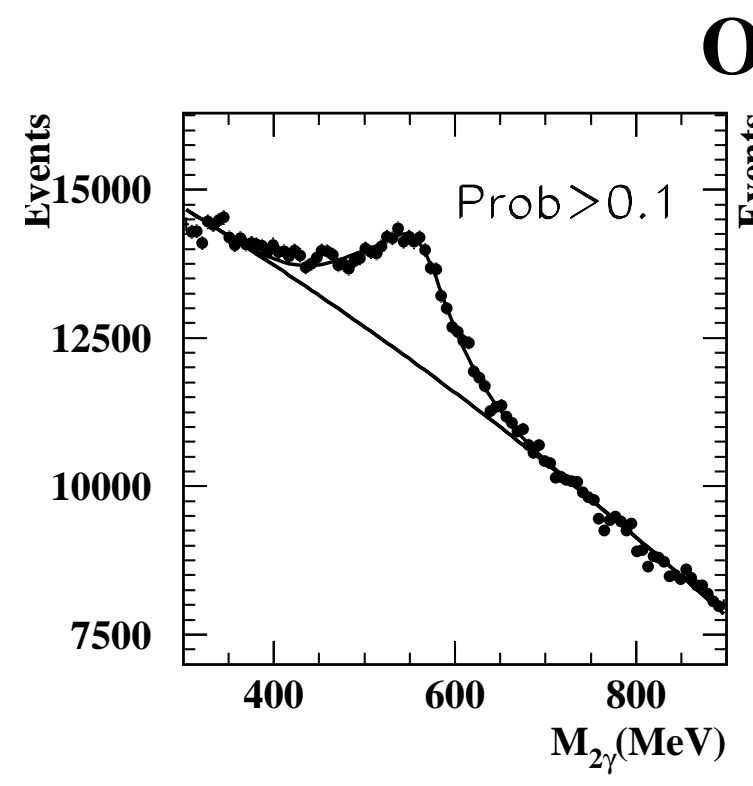

OPAL
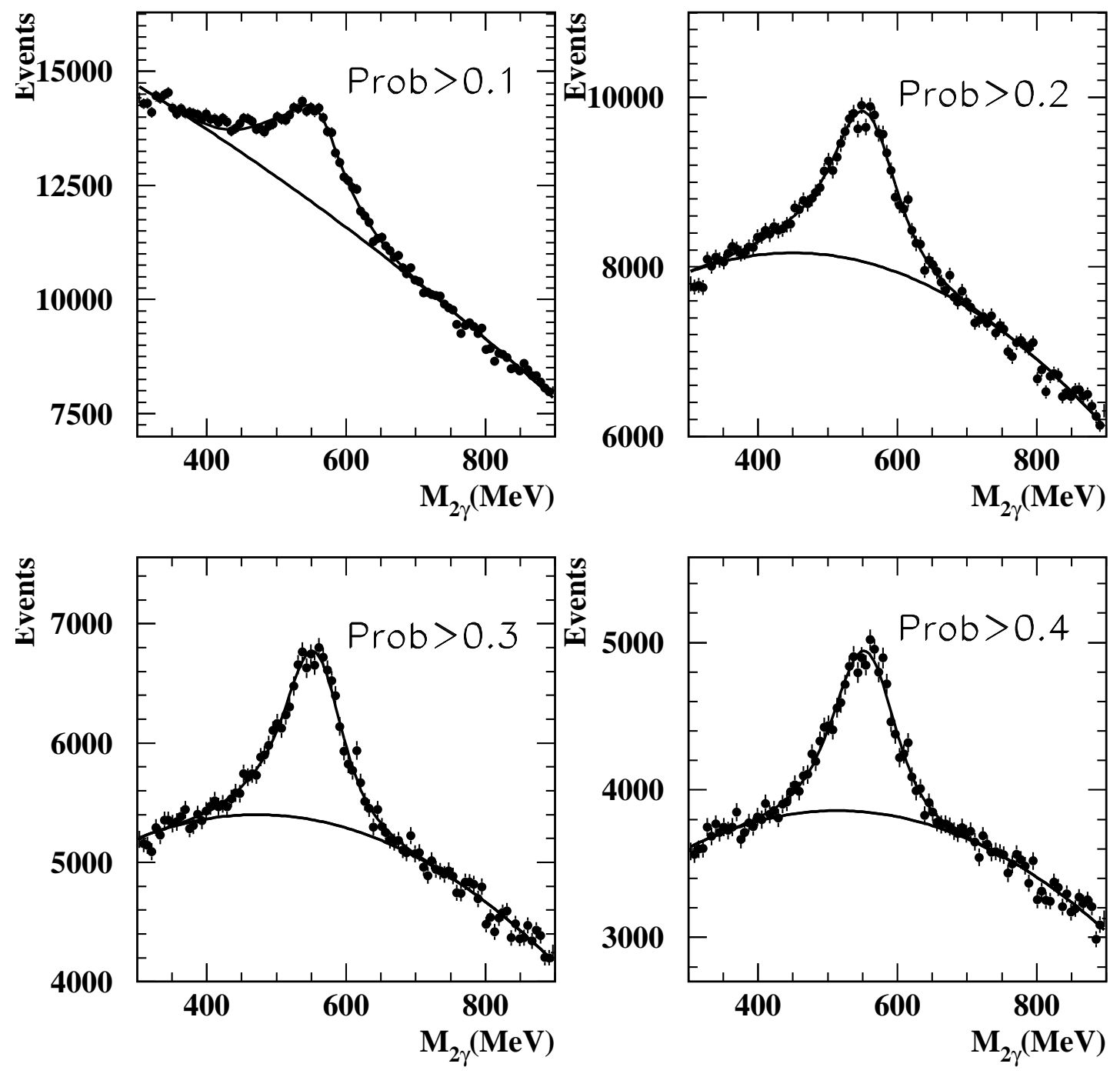

Figure 6: Two-photon invariant mass distribution in the vicinity of the $\eta$ for different cuts on the probability (prob) $\mathrm{P}=\mathrm{W}_{1} \times \mathrm{W}_{2}$. The smooth curve is from the fit to a double Gaussian and a second order polynomial. Note the suppressed zero on the vertical axis. 


\section{OPAL}

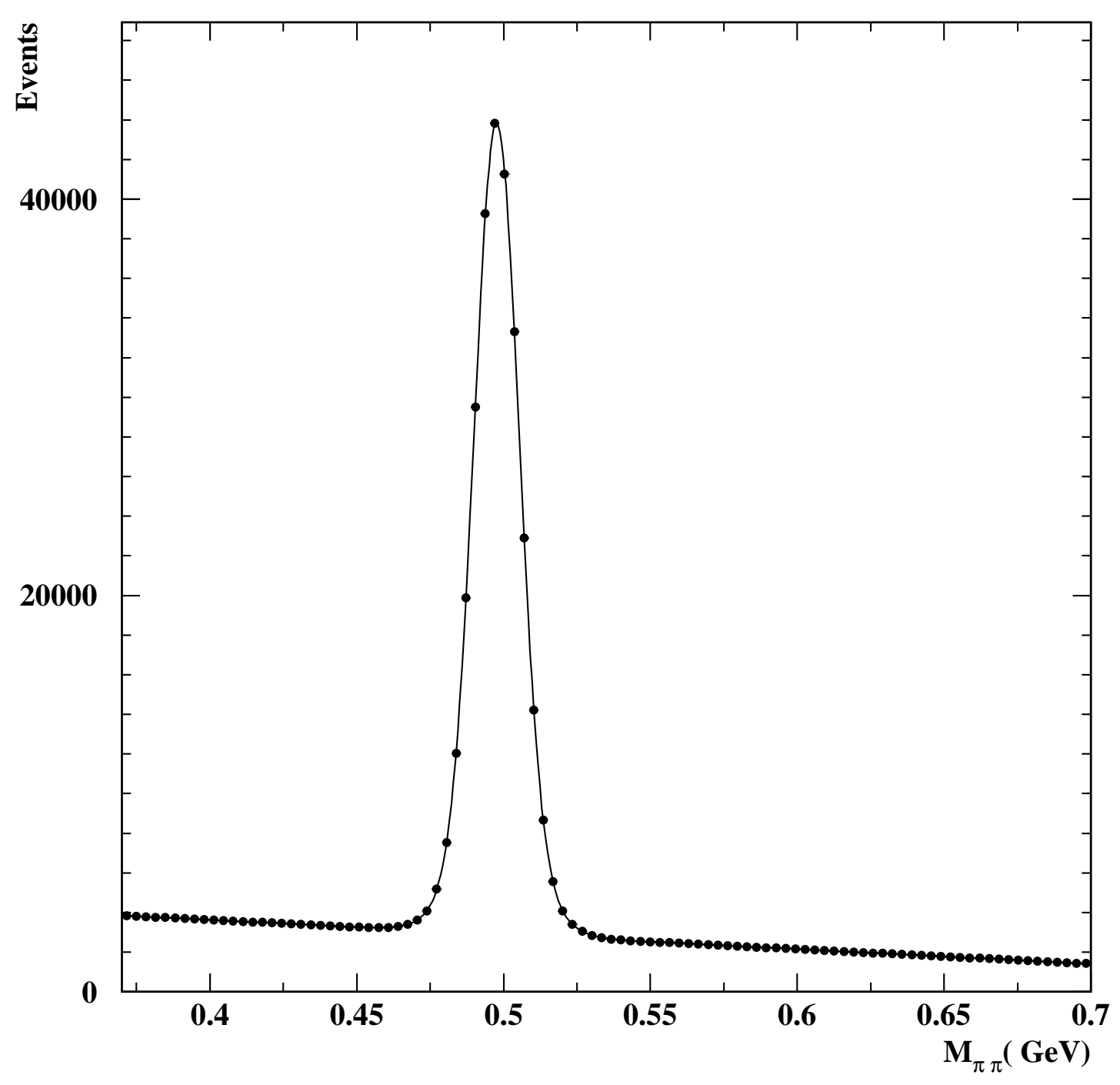

Figure 7: $\pi^{+} \pi^{-}$invariant mass distribution in the vicinity of the $\mathrm{K}^{0}$. The smooth curve is from the fit to a double Gaussian and a second order polynomial. 


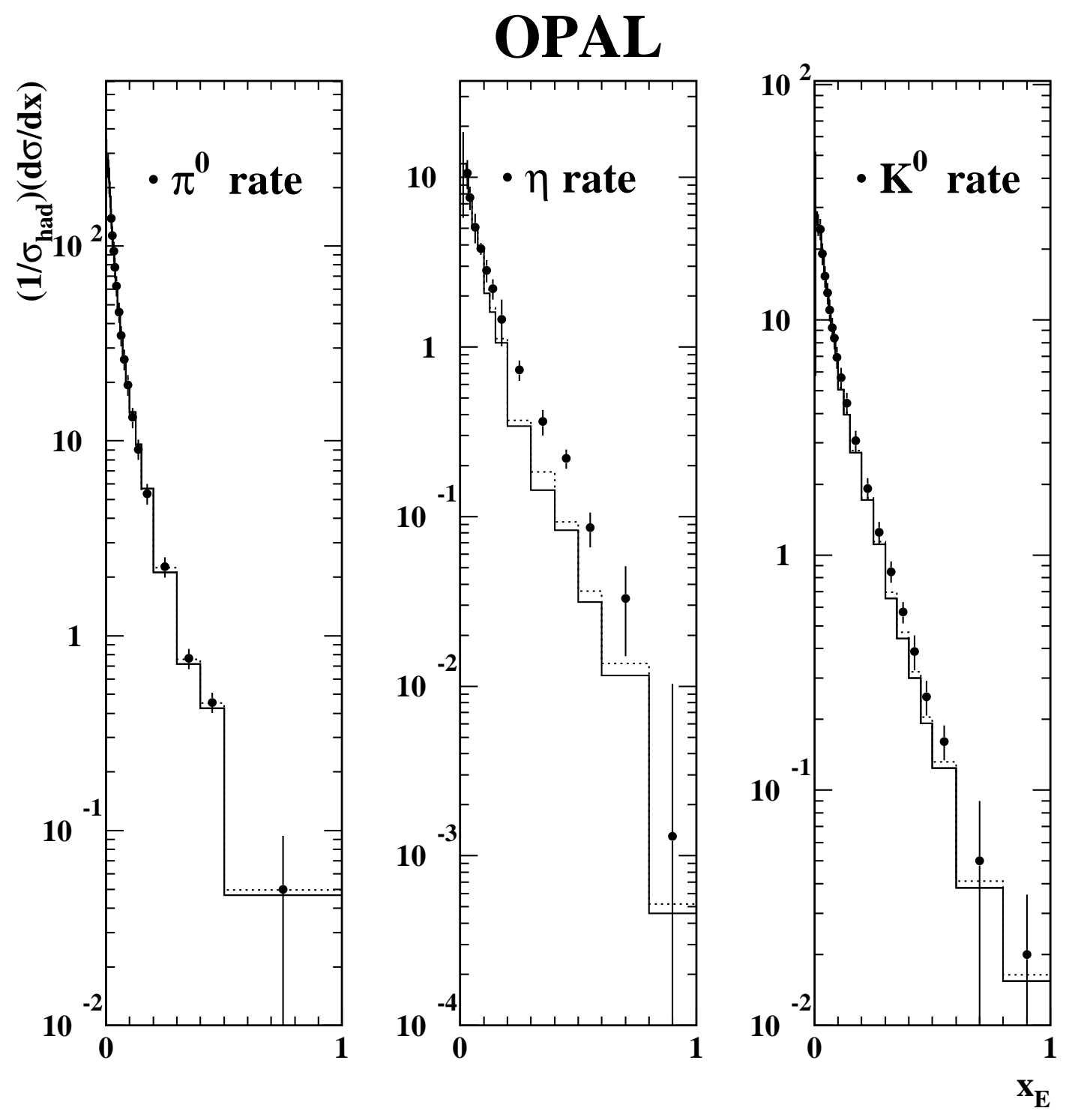

Figure 8: Differential rates of $\pi^{0}, \eta$ and $\mathrm{K}^{0}$ in $\mathrm{Z}^{0}$ hadronic decays. The errors for the data points include the systematic errors. The histograms and the dotted lines are from Jetset and Herwig Monte Carlo expectations respectively. 
OPAL
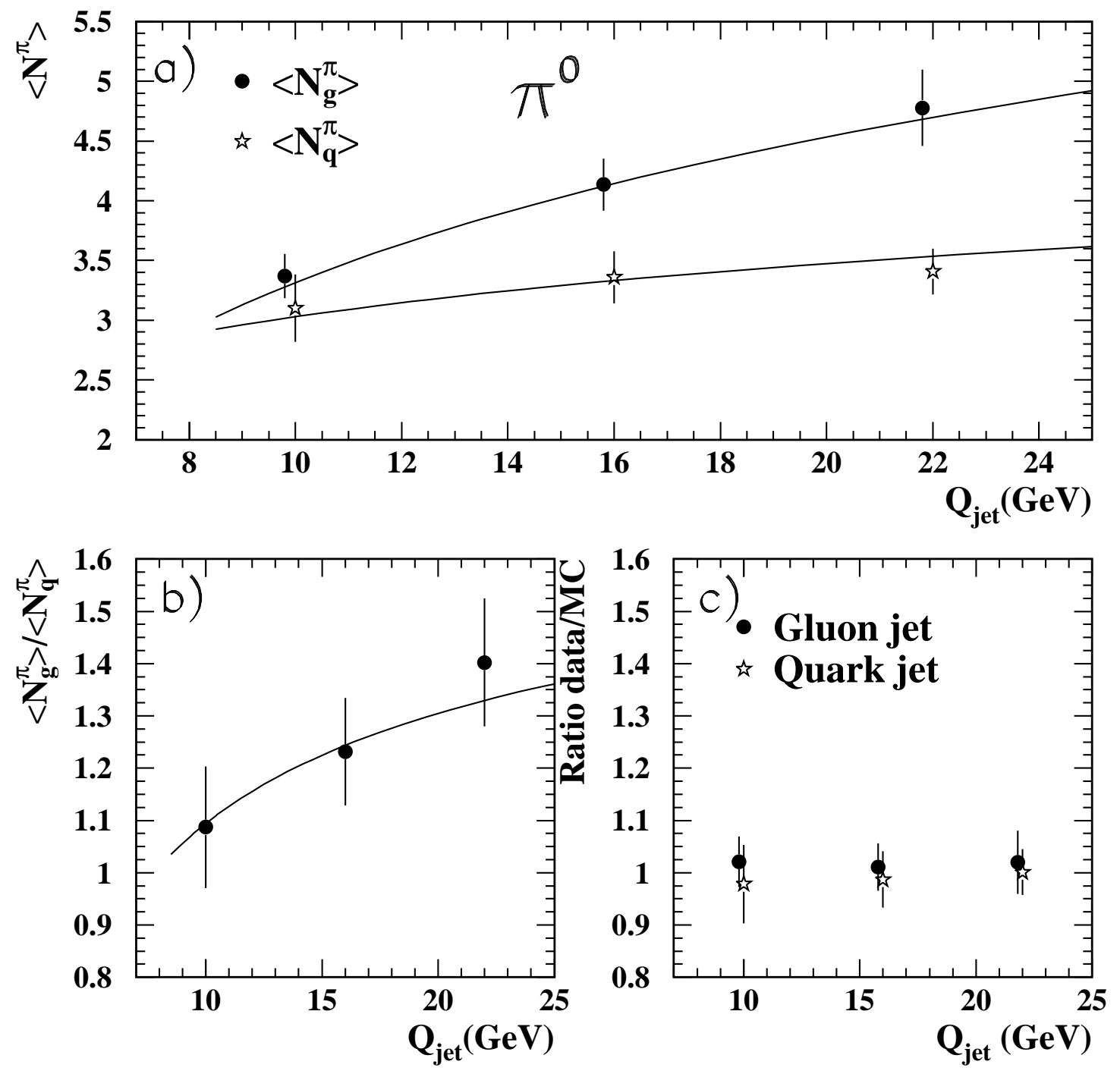

Figure 9: a) Average number of $\pi^{0}$ produced in pure gluon and quark jets as a function of the scale $Q_{\text {jet }}$. The curves are the functions obtained for the average charged particle multiplicity scaled by a normalisation factor of 0.47 . b) Ratio of production of $\pi^{0}$ in gluon and quark jets, as a function of $\mathrm{Q}_{\mathrm{jet}}$. The curve is the ratio of the functions obtained for the average charged particle multiplicity in gluon and quark jets. c) Ratio of production rate of $\pi^{0}$ in the data to that in the Jetset Monte Carlo, for gluon and quark jets. The gluon data points are shifted to the left for clarity. Error bars include systematic and statistical errors added in quadrature. 
OPAL
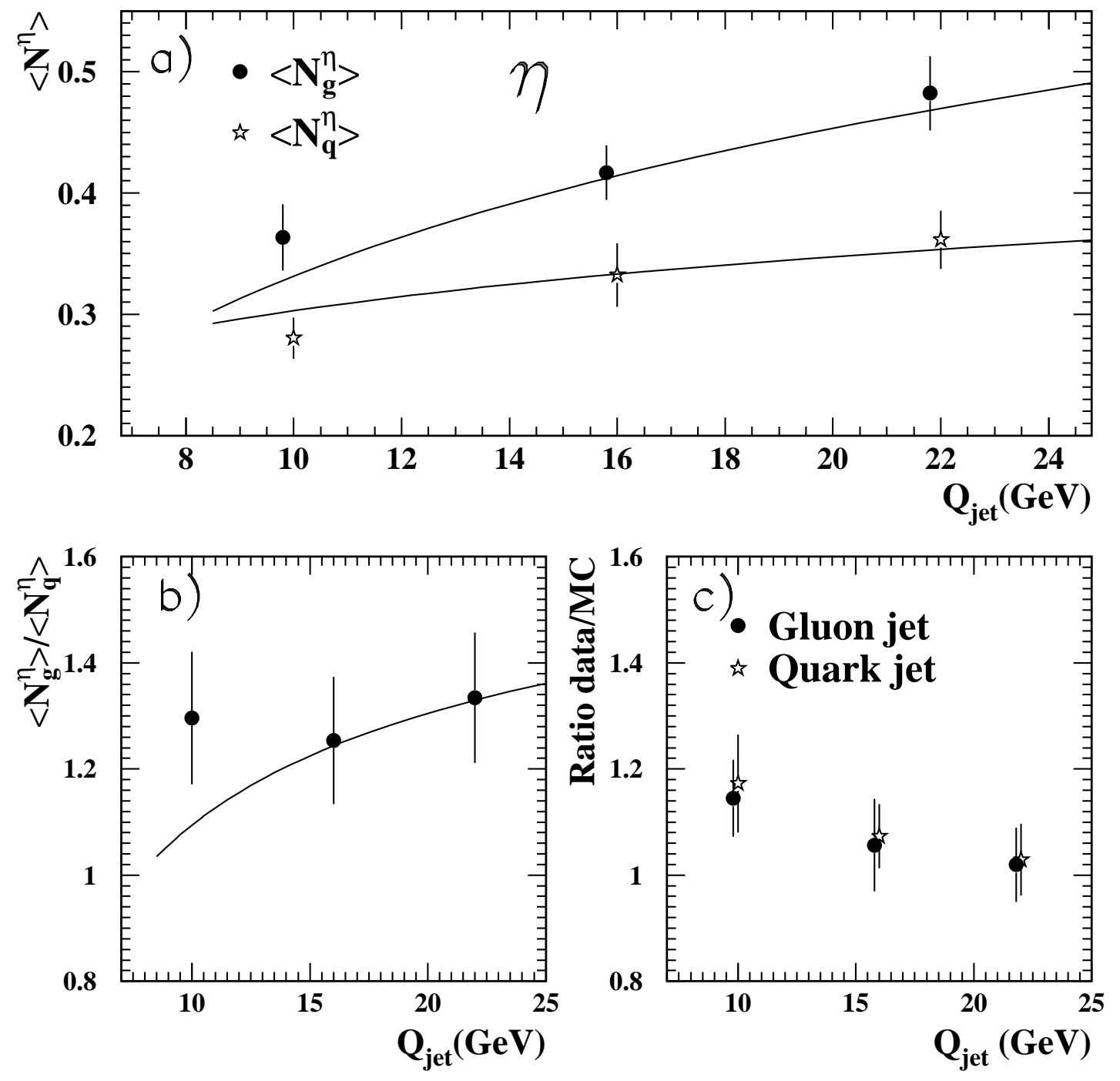

Figure 10: a) Average number of $\eta$ produced in pure gluon and quark jets as a function of the scale $Q_{\text {jet }}$. The curves are the functions obtained for the average charged particle multiplicity scaled by a normalisation factor of 0.047 . b) Ratio of production of $\eta$ in gluon and quark jets, as a function of $\mathrm{Q}_{\mathrm{jet}}$. The curve is the ratio of the functions obtained for the average charged particle multiplicity in gluon and quark jets. c) Ratio of production rate of $\eta$ in the data to that in the Jetset Monte Carlo, for gluon and quark jets. The gluon data points are shifted to the left for clarity. Error bars include systematic and statistical errors added in quadrature. 

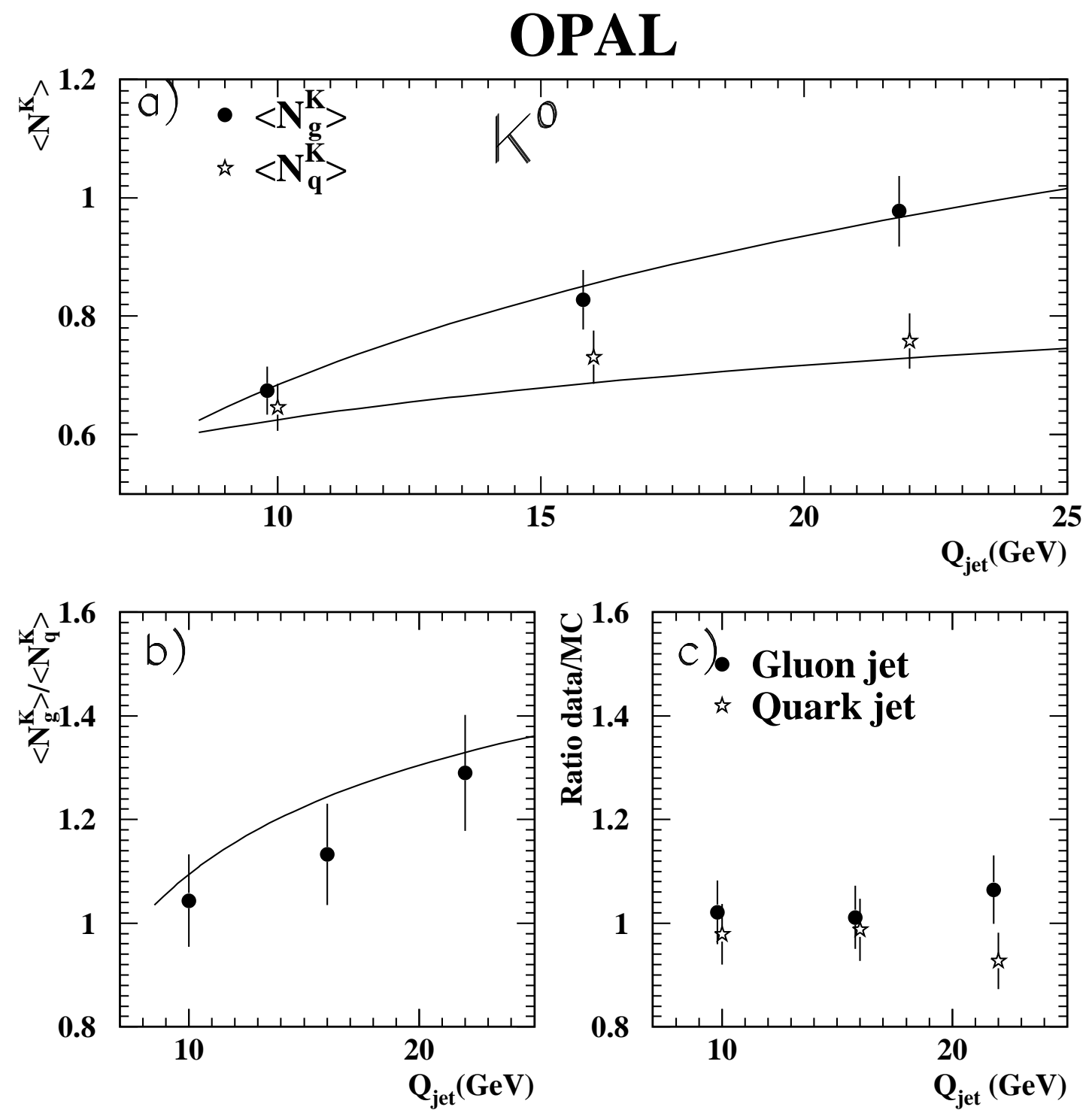

Figure 11: a) Average number of $\mathrm{K}^{0}$ produced in pure gluon and quark jets as a function of the scale $Q_{\text {jet }}$. The curves are the functions obtained for the average charged particle multiplicity, scaled by a normalisation factor of 0.097. b) Ratio of production of $\mathrm{K}^{0}$ in gluon and quark jets, as a function of $\mathrm{Q}_{\mathrm{jet}}$. The curve is the ratio of the functions obtained for the average charged particle multiplicity in gluon and quark jets. c) Ratio of production rate of $\mathrm{K}^{0}$ in the data to that in the Jetset Monte Carlo, for gluon and quark jets. The gluon data points are shifted to the left for clarity. Error bars include systematic and statistical errors added in quadrature. 
OPAL

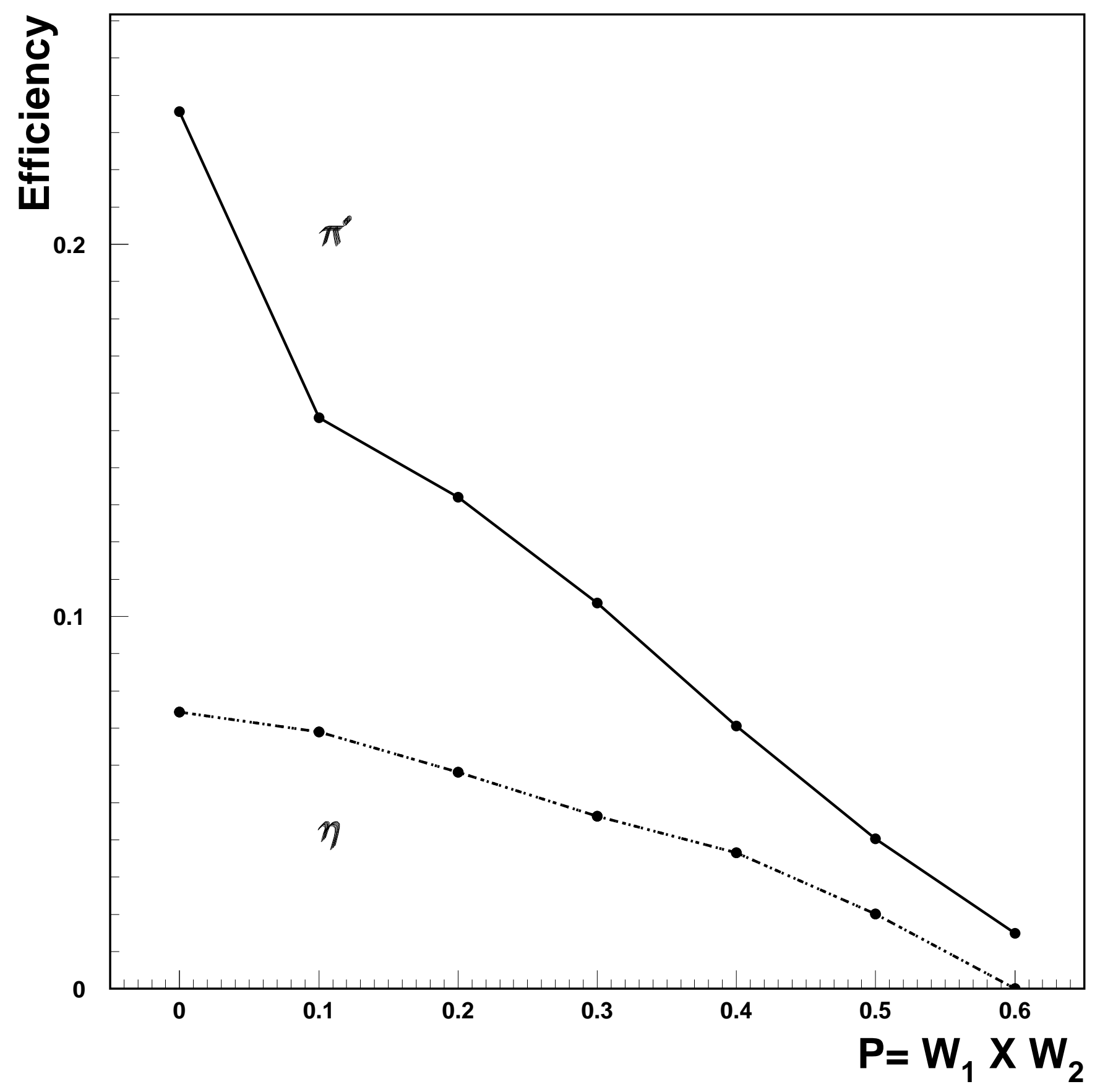




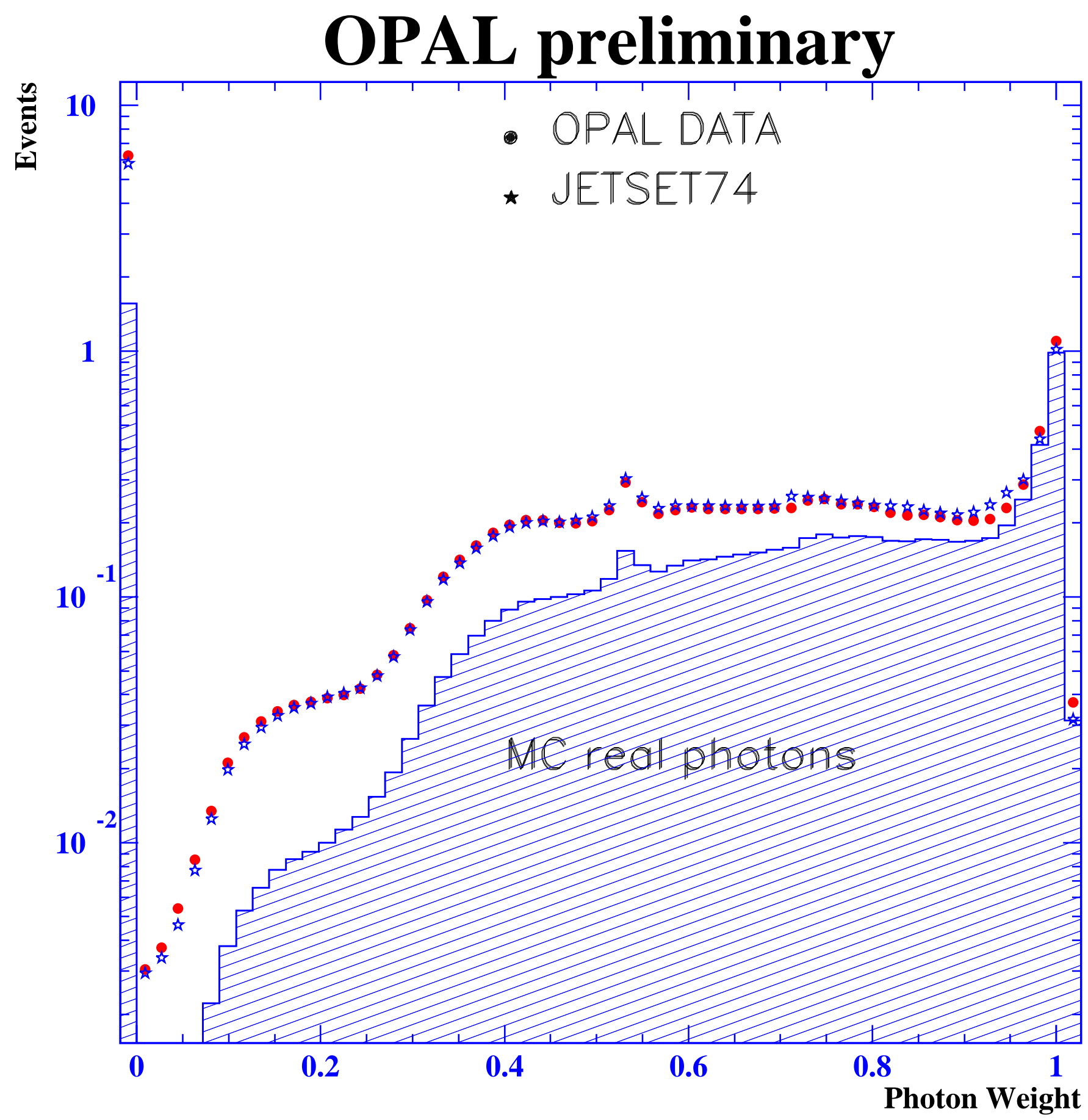

